\title{
Front propagation into unstable states. II. Linear versus nonlinear marginal stability and rate of convergence
}

\author{
Wim van Saarloos \\ AT\&T Bell Laboratories, Murray Hill, New Jersey 07974
}

(Received 21 December 1988)

\begin{abstract}
In an earlier paper, we developed a general physical picture for the linear-marginal-stability mechanism governing the dynamics of front propagation into linearly unstable states. The main conclusion from this approach and the expressions for the resulting front velocity are similar to those obtained along different lines for the space-time evolution of instabilities in plasma physics and fluid dynamics with the so-called pinch-point analysis (a special type of saddle-point analysis). However, as stressed by Ben-Jacob et al. [Physica 14D, 348 (1985)], it is known from the work of Aronson and Weinberger [in Partial Differential Equations and Related Topics, edited by J. A. Goldstein (Springer, Heidelberg, 1975); Adv. Math. 30, 33 (1978)] on a class of simple model equations that exceptions can occur to the linear-marginal-stability velocity selection. In this paper, we generalize these observations and incorporate such exceptions into our general picture of front propagation into unstable states. We show that a breakdown of linear marginal stability occurs if the linear-marginal-stability front profile becomes unstable against a particular nonlinear "invasion mode." If this happens, a larger front speed is selected at a point at which the front profile is now marginally stable against this nonlinear invasion mode. We therefore refer to this as the nonlinearmarginal-stability mechanism. (Ben-Jacob et al. call it case-II marginal stability.) We present the results of detailed numerical studies that support our identification of the nonlinear-marginalstability mechanism, and present the first examples of it for fronts in pattern-forming systems. In the neighborhood of a transition from linear to nonlinear marginal stability, the wavelength of the pattern generated by the front is only weakly dependent on the nonlinearities. We also analyze front propagation properties close to the threshold for instability at a pitchfork bifurcation. We conclude that linear marginal stability generally holds near a continuous transition (corresponding to a supercritical or forward bifurcation point), while front propagation close to a first-order transition (corresponding to a subcritical or inverted-bifurcation point) is generally governed by nonlinear marginal stability. These results are of importance for recent applications of the various approaches in fluid dynamics and other fields. Finally, we derive an expression for the rate of convergence of the front velocity to its asymptotic value. For the class of equations studied by Aronson and Weinberger, our expression reduces to a rigorous result by Bramson [Mem. Am. Math. Soc. 285, 1 (1983)], but it differs from the one often quoted in the pinch-point or saddle-point analysis. We argue that the latter one is only valid in a limited region of space, and show how to extend the usual analysis to arrive at our result. Several experimental systems to which our results are relevant are discussed.
\end{abstract}

\section{INTRODUCTION}

In the last few years, the problem of front propagation into unstable states has received renewed experimental and theoretical interest in the physics community. ${ }^{1-13}$ One class of systems that exhibits these types of fronts-a propagating region of space where the properties of the system vary sharply in a certain direction-is one whose time evolution, following a quench into an (absolutely) unstable state, is dominated by the propagation of welldeveloped fronts or domain walls separating the unstable state from some other state. Such behavior has, for example, been studied in fluid dynamics experiments on Taylor-Couette ${ }^{1,12}$ and Rayleigh-Bénard ${ }^{13}$ systems, in liquid crystals, ${ }^{14-16}$ and in a simple chemical wave experiment $^{17}$ (as discussed later, fronts in excitable media ${ }^{18,19}$ are different). Typically, such experiments need to be carefully controlled, since the state into which the front propagates is absolutely unstable, meaning that, viewed at a fixed position, perturbations are found to grow.

A second class of systems for which the study of the propagation of perturbations into an unstable state is relevant, consists of those that are convectively unstable. In this case, a perturbation not only grows in time but is also convected away, and this convection is strong enough to make the system locally stable. As a result, the long-term evolution of a particular convectively unstable system will depend on its size, the boundary conditions, and the presence of noise. ${ }^{20,21}$ Likewise, these factors will determine the extent to which the system exhibits well-developed fronts, by which we mean the (nonlinear) transition region between the convectively unstable state of the system and some other, usually stable, state of the system. However, the propagation of such fronts is governed in a number of cases by the same 
mechanism of growth and convection as the one controlling the linear perturbations, and therefore the study of front propagation is relevant for understanding convectively unstable systems as well. Clearly, since convectively unstable systems are stable locally ${ }^{22}$ (at a fixed position), they usually do not only occur under carefully prepared experimental conditions, but also arise naturally in various fields of physics in experimentally less wellcontrolled situations. While early theoretical studies of the linear time evolution of convectively and absolutely unstable states were done mostly in the field of plasma physics, ${ }^{22}$ their relevance to fluid mechanics ${ }^{21-25}$ and pattern selection ${ }^{26}$ - especially at the transition from a convective instability to an absolute instability ${ }^{26-28}$ or in relation to the sensitivity to noise $\mathrm{e}^{20,21}$ - has recently become recognized as well. Thus the study of front propagation into unstable states (convectively as well as absolutely) is an important ingredient for understanding a variety of problems.

It should be kept in mind that in this paper front propagation into an unstable state is always understood to mean front propagation into a linearly unstable state. Apart from the experiment by Hanna et al. ${ }^{17}$ on waves in an iodate arsenous acid system, most chemical waves do not fall in this class. The essential feature leading to traveling pulses and wave trains in excitable media ${ }^{18,19}$ is $b i$ stability, and the relevant fronts for these situations are those between two stable states. We will discuss the differences in some more detail in Sec. VII of this paper.

In this paper, I will mainly concentrate on the dynamical theory ${ }^{9}$ for front propagation into unstable states that leads to the marginal-stability picture first hypothesized by Dee, Langer, and co-workers. ${ }^{2,3}$ This theory ${ }^{9}$ is based on the intuitive idea that most properties of fronts propagating into an unstable state can be obtained from an analysis of the dynamical evolution in the leading edge of the front profile, i.e., the region where the deviations from the unstable state are small enough that the equations can be linearized. The formulation of my earlier paper, ${ }^{9}$ which builds on a reformulation and extension of some of the ideas of Dee and co-workers, ${ }^{2,3}$ and Shraiman and Bensimon, ${ }^{8}$ identifies the general properties of front propagation into unstable states that drive the velocity of initially localized fronts to a special value $v^{*}$, the so-called marginal-stability velocity. This name derives from the fact that at $v^{*}$, the front profile also changes stability (front profiles with velocity $v>v^{*}$ are stable, with $v<v^{*}$ unstable).

Another approach to the dynamical evolution of small perturbations around an unstable state was developed since the late 1950 s by several workers in plasma physics. ${ }^{22}$ The method, summarized elegantly in the book by Lifshitz and Pitaevskii, ${ }^{29}$ is based on an asymptotic analysis of the Green's function of the linearized evolution equation using contour deformation techniques for the inverse Laplace-Fourier transform. In the language appropriate to this formulation, ${ }^{22}$ the long-time behavior is determined by a "pinch point" in the complex plane, at which two roots of the dispersion relation "pinch off" the integration contour (the pinch point is a special type of saddle point). Besides differences in language between this approach and the marginal-stability theory, there is a difference in focus-in the pinch-point analysis, ${ }^{22}$ usually less attention is paid to the importance of initial conditions, and the asymptotic behavior for $t \rightarrow \infty, x$ fixed is derived; in the marginal-stability formulation, however, the requirement that initial conditions be sufficiently localized emerges naturally ${ }^{1-3,8,9}$ and the analysis focuses on the propagation of the leading edge moving with the front, i.e., the limit $t \rightarrow \infty, x \rightarrow \infty, x / t$ fixed. Nevertheless, in first order the equations for the pinch point are exactly the same as those for the marginal-stability point, and one can even translate the conditions arising in one formulation into the language of the other. These connections, as well as the relative merits of one formulation over the other, will be the theme of a future paper. ${ }^{30}$ As we shall see, however, the two approaches yield different expressions for the rate of approach to the asymptotic behavior, due to the different way in which the limits are taken. The limit $x, t \rightarrow \infty, x / t$ fixed considered in the dynamical approach is the natural one for front propagation.

Both the dynamical theory ${ }^{9}$ and the pinch-point formulation are based on an analysis of the linearized dynamical equations; henceforth, we will therefore, within the context of the dynamical approach, refer to $v^{*}$ as the linear-marginal-stability value. However, as BenJacob et al. ${ }^{3}$ point out, the work by Aronson and Weinberger ${ }^{1}$ shows that there are explicit examples of cases in which the fronts propagate with speeds $v^{\dagger}\left(>v^{*}\right)$ different from the linear-marginal-stability value $v^{*}$. For the particular type of equations considered by Aronson and Weinberger ${ }^{1}$ (first order in time, second order in space), Ben-Jacob et al. ${ }^{3}$ showed, however, that the existence of a different front speed $v^{\dagger}$ can also be related to a stability property of the fronts. For speeds $v<v^{\dagger}$, front solutions are unstable to an isolated mode of the fully nonlinear equations. While they refer to this situation as "case-II marginal stability," I will therefore call $v^{\dagger}$ the nonlinear-marginal-stability value.

It appears that one of the main advantages of the dynamical approach a $^{2,3,6,9}$ over the pinch-point analysis is that the condition under which the linear analysis breaks down is much more readily understood in the first approach. Indeed, except for a remark by Lifshitz and Pitaevskii, ${ }^{29}$ the importance of initial conditions is usually hardly mentioned in the pinch-point literature, while the analogue of nonlinear marginal stability has, to my knowledge, never been considered within this formulation. Since the most promising applications ${ }^{22-28}$ of the theory are to systems whose dynamics is certainly nonlinear behind the front, a proper understanding of these effects is quite important.

That nonlinear marginal stability has physical relevance and is not a pathological mathematical exception, is illustrated by the chemical wave experiment of Hanna et al. ${ }^{17}$ The wave fronts they study propagate with the nonlinear-marginal-stability speed $v^{\dagger}$, which is much larger than the value of $v^{*}$. Also, recent experiments by Cladis et al. ${ }^{31}$ on wall motion in smectic- $C^{*}$ liquid crystals in an electric field are believed to show a crossover from a linear-marginal-stability regime to a 
nonlinear-marginal-stability regime with increasing field strength. Although I will concentrate in this paper on the general mechanism of front propagation, I will show in Sec. $X$ with several examples that the work in this paper is experimentally much more relevant than one might guess on the basis of just these two examples. Indeed, as we will discuss, nonlinear marginal stability generally occurs near subcritical bifurcations, and the distinction between linear and nonlinear marginal stability sheds new light on several fundamental instabilities in fluid dynamics.

In my earlier paper ${ }^{9}$ on the dynamical theory of linear marginal stability, the exceptions arising from nonlinear marginal stability were not discussed in detail; remark (4) about it in Sec. IV is actually incorrect. In this paper, we will show that nonlinear marginal stability can be readily implemented into our formulation. Indeed, a reformulation of the arguments presented by Ben-Jacob et al. ${ }^{3}$ for the specific equation they studied gives a very simple intuitive picture for the origin of nonlinear marginal stability. While our approach may be viewed as an immediate extension of the exact results for equations that are of first order in time and of second order in space, we especially focus on the underlying dynamical mechanism and its generality that allows us to apply it to more complicated cases. In particular, for arbitrary problems whose relevant fronts uniformly translate solutions of the type $\phi(x-v t)$, our approach allows us to predict whether or not nonlinear marginal stability occurs from an analysis of the nonlinear profiles $\phi(x-v t)$. This will be illustrated with an explicit example of a fourth-order partial differential equation. We will also present evidence that the same mechanism governs the transition to nonlinear marginal stability for pattern-forming fronts. However, without an explicit analysis of the nonlinear timedependent problem, our approach is not yet powerful enough to predict explicitly whether nonlinear marginal stability occurs in a given pattern-forming problem at arbitrary values of the parameters. Fortunately, near the instability threshold of a pattern-forming system, the dynamics often reduces to that of an amplitude equation for which the essential front propagation behavior is known, and this enables us to make explicit predictions in the physically most relevant regime.

The rate of approach to the asymptotic front velocities $v^{*}$ and $v^{\dagger}$ has not received much attention, neither in the pinch-point formalism nor in the marginal-stability theory. In this paper, we also show that this rate of approach can be derived easily with the dynamical theory. ${ }^{9}$ For linear marginal stability, we get an algebraic approach $v(t) \approx v^{*}-3 /\left[2\left(k^{i}\right)^{*} t\right]$, with $\left(k^{i}\right)^{*}$ the spatial decay rate of the profile. Specified to the FisherKolmogorov equation, this expression agrees with the result derived rigorously by Bramson, ${ }^{32}$ but it differs from the usual pinch-point (saddle-point) expression. For nonlinear marginal stability, we argue that the velocity relaxes exponentially fast in time, $v(t) \simeq v^{\dagger}=D e^{-\Delta t}$, and show that this is indeed found in numerical simulations.

The plan of this paper is as follows. In Sec. II, we first summarize the essentials of the dynamical theory developed earlier. ${ }^{9}$ We then discuss in Sec. III the excep- tion due to nonlinear marginal stability in the context of the dynamical approach, and give two examples of it in Sec. IV. The numerical results in support of our picture are presented in Sec. V. In Sec. VI we discuss the implications for the behavior close to bifurcation points. We then briefly sketch the main differences between the type of fronts we consider here and those arising in excitable media in Sec. VII, while in Sec. VIII we turn to an analysis of the rate of convergence. After we point out some simplifications of the theory ${ }^{9}$ in Sec. IX, we discuss in Sec. $X$ the relevance of this work to fluid dynamics and other fields.

\section{SUMMARY OF THE DYNAMICAL APPROACH}

The propagation of a front into an unstable state differs significantly from the well-known propagation of an interface or front into a metastable state. When a system in a metastable state is slightly perturbed, it relaxes back to that state; this implies that for a phase transformation to occur, fluctuations have to be pushed over some barrier. Accordingly, the speed of an interface propagating into a metastable state is determined by a balance between the driving force and the dissipation or the kinetic barrier in the nonlinear interfacial region. In the picture underlying the dynamical approach, ${ }^{9}$ front propagation into unstable states is very different: since virtually any small perturbation around the unstable state will grow out by itself, most of the important front dynamics already occurs in the leading edge of the front, where the deviations from the unstable state are small and described by the linearized equations. The dynamics of the nonlinear region of the front often just follows, as it were, that dictated by the dynamics in the leading edge of the profile.

Suppose we want to determine the front propagation into an unstable state $\phi=0$ of some field $\phi$. For long times, the profile in the leading edge will become of form $\phi \sim e^{-i \omega t+i k x}$, with $\omega(k)$ given by the linear-dispersion relation. To facilitate the comparison with the literature on space-time evolution of instabilities, ${ }^{22}$ we have included a factor $i=\sqrt{-1}$ in the exponent. This factor was not included in Ref. 9; formulas from that paper have to be transcribed by putting $k_{\text {here }}=i k_{9}$ and $\omega_{\text {here }}=i k_{9}$, i.e., the real parts of quantities in Ref. 9 become the imaginary part here. The definition of all other complex quantities has been changed likewise. To study the approach to the asymptotic form $e^{-i \omega t+i k x}$ and the selection of the relevant $k^{*}$ and $\omega\left(k^{*}\right)$, it is, as before, ${ }^{9}$ advantageous to transform to a complex field $u$ defined by $\phi(x, t)=e^{i u(x, t)}$. The spatial derivative $q=\partial u / \partial x$ of $u$ clearly plays the role of a local wave number $k$ and the derivative $-u_{t}$ that of the local growth rate $\omega$, and hence both these derivatives are expected to approach constant values for long times [note that $\omega$ and $k$, and hence $u$, can be complex: the imaginary part of $k$ is associated with the decay of the envelope and the real part with the oscillations, since $\left.\operatorname{Re} e^{i k x}=e^{-\operatorname{Im} k x} \cos (\operatorname{Re} k x)\right]$. Before specifying the dynamical equation that shows how and under what conditions the local wave vector $q$ and the velocity $v$ are driven towards the linear-marginal-stability values $k^{*}$ and $v^{*}$, it is useful to first summarize our intuitive under- 
standing of the dynamics in the leading edge, based only on our insight into the behavior of profiles consisting of a sum of terms of the form $e^{-i \omega t+i k x}$.

Since $k$ in $\phi \sim e^{-i \omega t+i k x}$ is in general complex, we have to analyze the dynamical selection of both its real and imaginary parts. The selection of a particular "mode" $\mathrm{Re} k$ can be understood as follows. For fixed values of $k^{i} \equiv \operatorname{Im} k$, the growth rate $\operatorname{Im} \omega(k)$ as a function of $k^{r} \equiv \operatorname{Re} k$ will have a maximum at some value of $k^{r}$, as sketched in Fig. 1 (the difference between the cases in which the maximum is at $k^{r}=0$ and $k^{r} \neq 0$ is discussed in Ref. 9, p. 222, and in Ref. 10). Thus, if we consider a superposition of profiles of the form $e^{-i \omega t+i k x}$ with all the same value of $k^{i}(=\operatorname{Im} k$ ), so that the spatial decay rate of the envelope is the same, the long-time appearance of the profile will be dominated by the mode $k^{r}$ corresponding to the maximum growth rate $\omega^{i}=\operatorname{Im} \omega$, i.e., for which $\partial \omega^{i} / \partial k^{r}=0$ and $\partial^{2} \omega^{i} /\left(\partial k^{r}\right)^{2}<0$. For understanding the long-time dynamical selection of a value of $k^{i}$ governing the spatial falloff of the envelope, it therefore suffices to consider for each value of $k^{i}$ only the maximum growth rate mode-this amounts to taking $k^{r}$ as an implicit function of $k^{i}$ through the requirement $\partial \omega^{i} / \partial k^{r}=0$. For these maximum-growth-rate profiles of the form $e^{-i \omega t+i k x}$, the envelope velocity $v=\omega^{i} / k^{i}$ is thus a function of $k^{i}$ only. Since for propagation into an unstable state the maximum growth rate $\omega^{i}$ must be positive in the limit $k^{i} \rightarrow 0$ (the "profile" then approaches a spatial Fourier mode), $v\left(k^{i}\right)$ diverges for $k^{i} \rightarrow 0$. Thus $v\left(k^{i}\right)$ is a decreasing function of $k^{i}$, and we will assume that $v\left(k^{i}\right)$ is of the form sketched in Fig. 2, with a minimum at some value $\left(k^{i}\right)^{*}$. Since the asymptotic spatial decay for a given velocity $v$ will be given by the smallest value of $k^{i}$, we will initially concentrate on the branch of solutions corresponding to the smallest values of $k^{i}$, indicated by the solid line.

In reality, the envelope of a front will, of course, be smooth. However, to bring out the essence of the dynamical mechanism, it is easier to imagine a hypothetical front consisting of two pieces of the form $e^{-i \omega t+i k x}$ with different values of $k^{i}$ (governing the spatial decay) and velocity $v\left(k^{i}\right)=\omega^{i} / k^{i}$. As sketched in Fig. 3, the dashed piece drops off slower than the piece drawn with a solid line $\left(k_{A}^{i}<k_{B}^{i}\right)$, but in agreement with the behavior of the

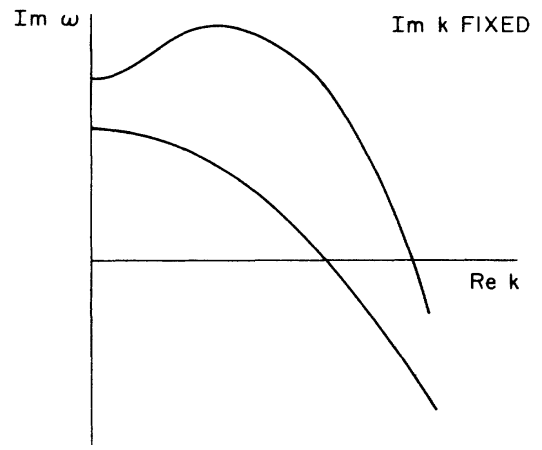

FIG. 1. Two types of possible behavior of the growth rate $\omega^{i}$ as a function of $k^{r}$ for $k^{i}$ fixed. For front propagation into an unstable stable, the maximum values of $\omega^{i}$ will be positive for relevant values of $k^{i}$.

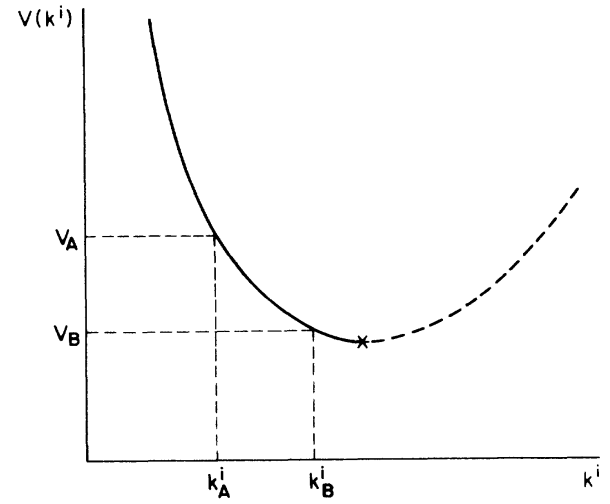

FIG. 2. Typical behavior of $v\left(k^{i}\right)$ for front propagation into an unstable state. In this plot $k^{r}$ is an implicit function of $k^{i}$ through the requirement $\partial \omega^{i} / \partial k^{r}=0$. The behavior of the fronts corresponding to points $A$ and $B$ is shown in Fig. 3.

function $v\left(k^{i}\right)$, the profile $A$ also moves faster than $B$. Nevertheless, as the figure demonstrates, the slowest moving part of the profile expands in time, i.e., becomes dynamically dominant. It is clear from Fig. 3 that this property is due to both (i) the fact that the fastest of the two profiles has the slowest spatial decay, and (ii) the fact that the part with the fastest spatial decay is to the right of the one with the slower spatial decay. Regarding (i), the connection between slope and velocity, note that by

(a)

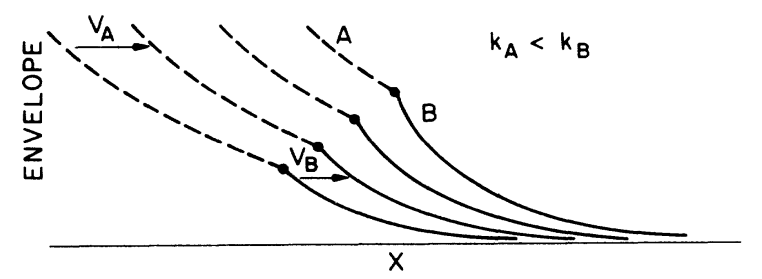

(b)

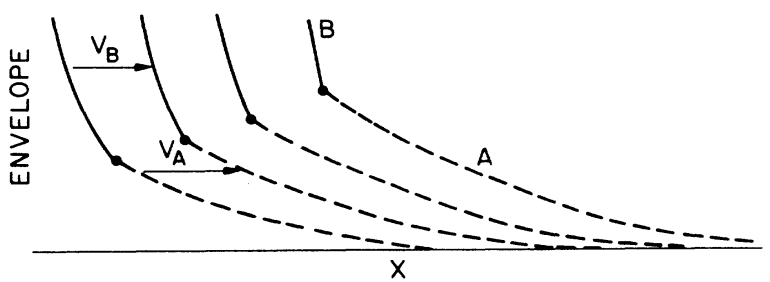

FIG. 3. Intuitive illustration of velocity selection. (a) The lower part of the envelope of some front profile, drawn with a solid line, corresponds to point $B$ in Fig. 2. It moves slower than the dashed part (corresponding to point $A$ in Fig. 2), but falls off steeper. The figure illustrates how the crossover point moves up in time, so that the profile becomes more and more dominated by the slowly moving part. This is a result of the decrease of the velocity with the slope of the profile, i.e., the fact that $v_{A}>v_{B}$ while $k_{A}<k_{B}$. (b) If the slowest profile $B$ is not the one to the right, the fastest profile emerges. Thus the selection illustrated in (a) only occurs if the initial conditions are sufficiently localized. 
taking $k_{A}^{i}$ close to $k_{B}^{i}$, one may easily convince oneself that the slower profile generally emerges as long as $d v\left(k^{i}\right) / d k^{i}<0$. As argued above, the negative slope of $v\left(k^{i}\right)$ is a general feature of front propagation into unstable states. Thus, if we imagine a smooth front as a collection of pieces with different values of $k$, this simple picture immediately suggests that the front velocity will continue to slow down as long as $d v / d k^{i}<0$ (near $v^{*}$, the asymptotics is actually somewhat more complicated, and $v$ approaches $v^{*}$ from below; see Sec. VIII). Observation (ii) above, viz., the fact that the slowest profile dynamically dominates because it is in front of the faster one is further illustrated in Fig. 3(b). Here, we show that if the two pieces are interchanged, so that the faster one is to the right, the faster one dominates the long-time dynamics. These observations imply that initial conditions are important: only if the initial profile $\phi(x, t=0)$ drops off faster than $e^{-\left(k^{i}\right)^{*} x}$ will the slowing down of the velocity continue all the way to the minimum value $v^{*}$ on the curve. Unless stated otherwise, we will henceforth always assume that the initial conditions are sufficiently localized, i.e., fall off faster than $e^{-\left(k^{i}\right)^{*} x}$. In this case, therefore, the above argument shows that $v^{*}$ will be the asymptotic front velocity. From the definition of $v\left(k^{i}\right)=\omega^{i} / k^{i}$ with $\partial \omega^{i} / \partial k^{r}=0$, it is easy to show that this minimum is given by ${ }^{2,3,9}$

$$
v^{*}=\frac{\omega^{i}}{k^{i}}=\frac{\partial \omega^{i}}{\partial k^{i}}, \quad \frac{\partial \omega^{i}}{\partial k^{r}}=0 .
$$

These equations determine the linear-marginal-stability velocity $^{2,3,9} v^{*}$ as well as the wave number $k^{*}$ at that point. As mentioned earlier, the name marginal stability refers to the fact that the point defined by Eq. (2.1) is also the point at which the stability of the front profiles changes. Indeed, the quantity $\partial \omega^{i} / \partial k^{i}$ (with $\partial \omega^{i} / \partial k^{r}=0$ ) plays the role of a group velocity $v_{\mathrm{gr}}$ for small perturbations in the local value of $k^{i}$. We can think of the effect of virtually all perturbations that decay faster than the envelope of a front traveling at speed $v>v^{*}$ as giving rise to a small change in the local wave number $k^{i}$ in the leading edge. Since on the branch drawn with a solid line in Fig. $2 v_{\mathrm{gr}}<v$, the front profiles are linearly stable simply because the front out runs the perturbation. ${ }^{9}$ Equation (2.1) therefore locates the point where $v_{\mathrm{gr}}=v$, i.e., where fronts are marginally stable as perturbations just keep up with them. As we shall discuss in Sec. III, in some cases an exceptional type of perturbation can nevertheless render the profiles unstable for velocities $v \geq v^{*}$; this will correspond to the nonlinear-marginal-stability scenario.

The above intuitive line of reasoning is supported by a more precise analysis of the leading-edge dynamics of smooth front profiles. For concreteness, we only summarize here the results ${ }^{9}$ for front propagation in systems described by a partial differential equation that is of first order in time but of arbitrary order in the spatial derivatives. In Sec. VIII, where we discuss the long-time convergence in more detail, we will show that the analysis is essentially unaltered for equations that include higherorder time derivatives.

For the systems under consideration, substitution of $\phi=e^{i u(x, t)}$ into the linearized equation leads to a dynami- cal equation of the form $u_{t}=-f\left(q, q_{x}, \ldots\right)$, where $q=\partial u / \partial x$ is the local wave number. To obtain a dynamical equation for $q$, it is most convenient to write $q$ as a function of $u^{i}=\operatorname{Im} u$. Since $u^{i}$ is a measure of the envelope of the front, this amounts to a transformation to a comoving frame. For $q\left(u^{i}, t\right)$, we then obtain [cf. Ref. 9, Eq. (3.21)]

$$
q_{t}=\left(\frac{f^{i}}{q^{i}}-f_{q}\right] q^{i} q_{u}+\mathcal{L} q .
$$

Here the subscript $u$ denotes a differentiation with respect to $u^{i}$, and $\mathcal{L} q$ stands for ${ }^{33}$

$$
\begin{aligned}
\mathcal{L} q \equiv-q^{i} f_{q_{u}}\left(q, q_{u}, q_{u u}, \ldots\right) q_{u u} & -q^{i} f_{q_{u}}\left(q, q_{u}, q_{u u}, \ldots\right) \\
& \times q_{u u u}-\ldots .
\end{aligned}
$$

The behavior of Eq. (2.2) is consistent with the dynamical picture discussed earlier. Indeed, we can make contact with the earlier analysis by noting that for slowly varying $q\left(u^{i,} t\right)$, the terms $\mathcal{L} q$ are small, while $f^{i}\left(q, q_{u}, \ldots\right)$ $\approx f^{i}(q, 0,0, \ldots)=\left.\omega^{i}(k)\right|_{k=q}$. Thus the first term $f^{i} / q^{i} \approx \omega^{i} / k^{i}$ is essentially the envelope velocity $v$ and $\operatorname{Re} f_{q} \approx \partial \omega^{i} / \partial k^{i}$ is essentially the group velocity. With these identifications, it is then easy to demonstrate ${ }^{9}$ that the dynamical mechanism envisioned earlier is contained in the first term on the right-hand side, and that indeed for localized initial conditions the front velocity is driven towards the linear-marginal-stability value. In Sec. VIII, we will also derive the long-time asymptotics from this equation.

\section{NONLINEAR MARGINAL STABILITY}

The analysis sketched above, which shows why the front speed approaches the linear-marginal-stability value, is based on the assumption that the dynamically relevant branch $v\left(k^{r}\right)$ is the one drawn with a solid line in Fig. 2, i.e., the one corresponding to the smallest root $k^{i}$ solving the equations

$$
v=\frac{\omega^{i}}{k^{i}}, \quad \frac{\partial \omega^{i}}{\partial k^{r}}=0, \quad \frac{\partial^{2} \omega^{i}}{\left(\partial k^{r}\right)^{2}}<0 .
$$

The reason that we consider this branch the dynamically relevant one is that the asymptotic spatial decay $\phi \simeq C_{1}(v) e^{-i k_{1} x}+C_{2}(v) e^{-i k_{2} x}+\cdots$ will be dominated by the root with the smallest value of $k^{i}, k_{1}$ say, whenever the prefactor $C_{1}(v)$ of the exponential is nonzero. For uniformly translating profiles of the form $\phi(x-v t)$, a general counting argument ${ }^{9}$ does support this intuitive idea for arbitrary values of the velocity $v$. Nevertheless, it may happen that at some particular value $v^{\dagger}$, one has

$$
C_{1}\left(v^{\dagger}\right)=0 \text {, }
$$

so that the asymptotic spatial decay of the steady-state front profile is not given by the smallest root $k_{1}$ on (3.1), but by the next root $k_{2}^{i}$, with $k_{2}^{i}>k_{1}^{i}$. This situation is depicted in Fig. 4(a).

The dynamical implications of this can be understood immediately within the same picture as the one used before to understand linear marginal stability. Since, as il- 
lustrated in Fig. 4(b), the front moving at speed $v^{\dagger}$ drops off faster in space than any front profile with velocity $v \geq v^{*}$, this profile will overtake all other ones moving at smaller velocity, as Fig. 4(c) illustrates. As before, we have drawn the profiles with sharp breaks in the derivative, so as to bring out the effects more clearly, but again the dynamical selection illustrated in the figure is obviously valid quite generally: If there is a front profile with steady-state velocity $v^{\dagger}$, satisfying (3.2), then all fronts with velocity $v<v^{\dagger}$ will be unstable against "invasion" by the profile with asymptotic speed $v^{\dagger}$. This will lead to a breakdown of linear marginal stability, and we expect that the asymptotic speed of fronts emerging from

(a)

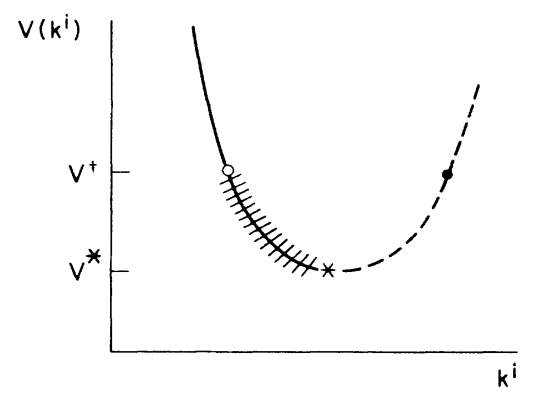

(b)

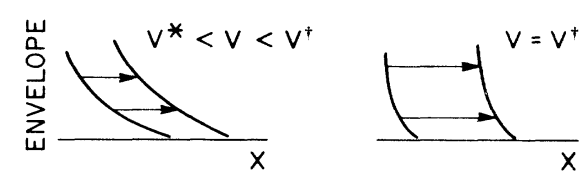

(c)

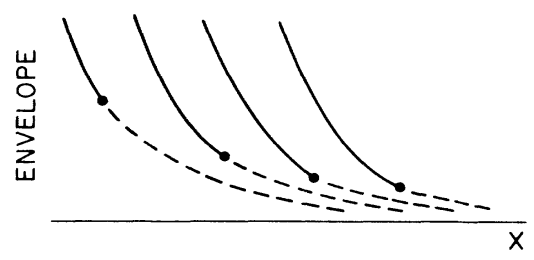

FIG. 4. Illustration of nonlinear marginal stability. (a) For all velocities $v \geq v^{*}$, the asymptotic spatial falloff of the envelope of some front profile is given by the values of $k^{i}$ determined by the solid line, except at the velocity $v^{\dagger}$. At this value, Eq. (3.2) holds and the asymptotic part is given by the value of $k^{i}$ corresponding to the dot. The solid line denotes the branch of solutions that is stable to perturbations; the cross-hatched line denotes the branch that is unstable to the "invasion mode." Except at $v^{\dagger}$, the dashed branch of $v\left(k^{i}\right)$ has no dynamical significance, since there are no front profiles whose asymptotic spatial decay is governed by these roots $k^{i}$. (b) Qualitative features of the front profiles corresponding to velocities $v^{*}<v<v^{\dagger}$ and $v=v^{\dagger}$. The profile with velocity $v^{\dagger}$ drops off fastest in space. (c) Illustration of velocity selection due to nonlinear marginal stability, in the same spirit as in Fig. 3. As a result of the behavior sketched in (b), front profiles corresponding to velocities $v<v^{\dagger}$ (dashed line) are unstable to an invasion by the profile moving with velocity $v^{\dagger}$. For long times, the relevant front velocity is therefore $v^{\dagger}$. sufficiently localized initial conditions becomes $v^{\dagger}$ instead of $v^{*}$. Both in the case of linear marginal stability and in the case of nonlinear marginal stability, the velocity selection of initially localized fronts can therefore, according to our picture, be summarized as follows: the selected front velocity is the one corresponding to the maximum value of $k^{i}$ that describes the asymptotic spatial falloff of a front profile.

Clearly, the existence of $v^{\dagger}$ is again connected with stability arguments, since all profiles with velocity $v<v^{\dagger}$ are unstable to the "invasion mode," while those with velocity $v>v^{\dagger}$ are stable [it is easy to convince oneself of this with a picture similar to Fig. 4(c)]. Thus $v^{\dagger}$ is also the velocity at which front profiles are marginally stable against this "invasion mode." However, while these dynamical implications can be understood completely within our picture of the leading-edge dynamics, the actual occurrence of a case in which the smallest root $k_{1}^{i}$ does not dominate the asymptotic spatial decay of a profile, depends on the properties of the whole front-it can only be determined by a global analysis including the leading edge as well as the nonlinear region behind it. We therefore refer to $v^{\dagger}$ as the nonlinear-marginal-stability value to distinguish it from the linear-marginal-stability value $v^{*}$, which can be calculated from a linear leading-edge analysis.

At first sight, one might wonder why the instability against the invasion mode was not automatically included in the earlier analysis summarized in Sec. II. The reason is that the arguments presented there and in Ref. 9 are based on the assumption that in the leading edge, $q_{i}$ is always smoothly varying with the velocity dependence $v\left(q^{i}\right)$ described approximately by the solid branch in Figs. 2 and 4(a). The invasion mode violates this assumption. Similarly, one should not conclude that the nonlinear-marginal-stability profile moving with $v^{+}$is unstable since $v_{\mathrm{gr}}>v$ on the dashed branch of the curves $v\left(q^{i}\right)$ in figs. 2 and 4(a): the spatial decay of the relevant perturbations does not correspond to smooth perturbations in $k^{i}$ along this dashed branch. Rather, the eigenfunctions corresponding to the relevant perturbations fall off with values of $k^{i}$ close to those on the solid branch at $v^{\dagger}$, and the nonlinear-marginal-stability profile is stable to these perturbations. See Appendix B for details.

Although the physical picture for nonlinear marginal stability is quite compelling, I am not able to demonstrate its validity in general. In particular, although I will show explicitly that nonlinear marginal stability can occur for envelope fronts-those whose dynamics remains intrinsically time dependent so that the front does not approach the uniformly translating type $\phi(x-v t)-\mathrm{I}$ do not know how to prove this mathematically. For equations whose relevant front solutions are uniformly translating profiles $\phi(x-v t)$, the change of stability at $v=v^{\dagger}$ can, however, be shown quite generally. This is discussed in Appendix A. For this class of fronts, the velocity selection rule formulated above also reduces to rigorous results derived by Aronson and Weinberger ${ }^{1}$ for equations of the type

$$
\phi_{t}=\phi_{x x}+F(\phi), \quad F(0)=0, \quad F^{\prime}(0)=1,
$$


and our picture is equivalent to the interpretation of these results discussed by Ben-Jacob et al. In fact, the rule formulated above may be viewed as the postulate that the velocity selection criterion ${ }^{1}$ derived for (3.3) holds quite generally.

Since the actual occurrence of nonlinear marginal stability in models more complicated than Eq. (3.3) is, at present, impossible to predict analytically except close to bifurcation points (see Sec. VI), it is useful to have an intuitive feeling for when one should expect to be in the nonlinear-marginal-stability regime. We therefore explore this in Sec. IV, before turning to a discussion of the numerical results.

\section{TWO EXAMPLES OF THE TRANSITION TO NONLINEAR MARGINAL STABILITY}

When is nonlinear marginal stability likely to occur? A general answer to this question cannot be given, but an example given by Ben-Jacob et al. ${ }^{3}$ is quite indicative, and so we will summarize it here.

Consider the equation

$$
\frac{\partial \phi}{\partial t}=\frac{\partial^{2} \phi}{\partial x^{2}}+\frac{\phi}{b}(b+\phi)(1-\phi) \text {. }
$$

This equation admits two homogeneous stable steady states, $\phi=1$ and $\phi=-b$, and one unstable state, $\phi=0$. When $b<1$, however, the state $\phi=-b$ is actually only metastable, and as a result a domain wall between this state and the absolutely stable state $\phi=1$ moves in the direction of the metastable state for $b<1$. (For $b>1$, the situation is reversed, since the equation is invariant under the transformation $b \rightarrow 1 / b, \phi \rightarrow-\phi / b$.) Its speed is ${ }^{3}$

$$
v_{w}=\frac{1-b}{(2 b)^{1 / 2}} .
$$

For $b$ close to 1 , front propagation into the unstable state of this equation is governed by linear marginal stability, and hence the front speed is $v^{*}=2$ [this value follows from applying Eq. (2.1) to (4.1)].

Consider now profiles that are initially of the form sketched in Fig. 5, in which the profile consists of a front

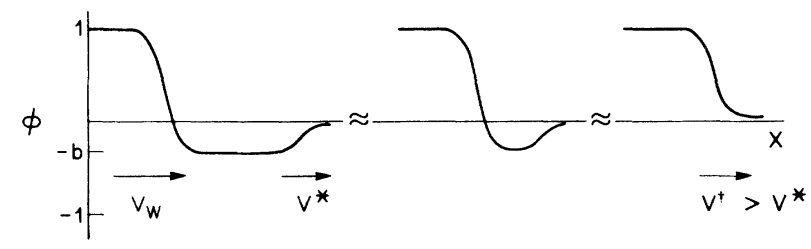

FIG. 5. Three stages in time of a front profile of Eq. (4.1), illustrating how nonlinear marginal stability can occur for strong asymmetry ( $b$ small). The initial profile consists of essentially two pieces: a first front between the unstable state $\phi=0$ and the metastable state $\phi=-b$ with speed $v \simeq v^{*}$, and a second one between $\phi=-b$ and the absolutely stable state $\phi=1$. When the latter wall travels faster, $v_{w}>v^{*}$, the leading edge is invaded by the second wall and the profile evolves to the situation on the right. There, the front propagates with speed $v^{+}>v^{*}$. between the unstable state $\phi=0$ and the metastable state $\phi=-b$, followed by a wall between $\phi=-b$ and the stable state $\phi=1$. When the two are far apart and $b$ is close to unity, the latter domain wall will, in view of (4.2), propagate slower than the initial front, and so the initial front will remain undisturbed. However, when we decrease $b$, the second domain wall will eventually overtake and destroy ${ }^{34}$ the initial front, since $v_{w} \rightarrow \infty$ for $b \rightarrow 0$. This suggests a crossover from linear marginal stability to nonlinear marginal stability for small enough $b$, because the second domain wall starts to play the role of the "invasion mode" envisioned earlier.

This is indeed what happens. For large $x$, a front $\phi(x-v t)$ propagating into the instable state $\phi=0$ with constant speed $v$, falls off as $\phi \simeq C_{1} e^{-K_{1} x}+C_{2} e^{-K_{2} x}$, with

$$
K_{1}=\frac{v-\left(v^{2}-4\right)^{1 / 2}}{2}, \quad K_{2}=\frac{v+\left(v^{2}-4\right)^{1 / 2}}{2} .
$$

However, as Ben-Jacob et al. ${ }^{3}$ point out, for

$$
v=(2 b)^{1 / 2}+(2 b)^{-1 / 2} \text {, }
$$

one can obtain the exact front solution

$$
\phi=\frac{1}{2}\left(1-\tanh \frac{q x}{2}\right),
$$

with

$$
\begin{array}{ll}
q=K_{1}, & \frac{1}{2}<b<2, \\
q=K_{2}, & 0<b<\frac{1}{2}, \quad b>2 .
\end{array}
$$

Clearly, for $0<b<\frac{1}{2}$, and $b>2$, these special solutions are just the nonlinear front profiles that satisfy (3.2) and so they locate exactly the values of $b$ where nonlinear marginal stability sets in. In other words, in this example, front propagation into the unstable state is, for $0<b<\frac{1}{2}$ or $b>2$, governed by nonlinear marginal stability with $v^{\dagger}$ given by (4.4), while for $\frac{1}{2}<b<2$ it is governed by linear marginal stability with $v^{*}=2$.

To put these findings into perspective, let us write the last term on the right-hand side of (4.1) as $\phi+\left(b^{-1}-1\right) \phi^{2}-\phi^{3} / b$. Thus we see that the effect of the nonlinear term $\phi^{3} / b$ is to decrease (locally) the growth rate $\partial \phi / \partial t$ with respect to the linear terms. For $b<1$, however, the $\phi^{2}$ term increases the local growth rate in regions where $\phi>0$. When $b^{-1}-1$ is large enough, this increase in the growth rate of the nonlinear region becomes so significant, that the dynamics in this region starts to drive the growth in the leading edge, and a transition to marginal stability occurs. In all examples I know of, the transition to nonlinear marginal stability is similarly related to an increase in the local growth rate of the nonlinear region.

For partial differential equations of second order, like those of the form (3.3) with a simple analytic form for $F(\phi)$, the nonlinear-marginal-stability velocity $v^{\dagger}$ can sometimes be calculated exactly. The reason is that the condition that the asymptotic decay of the profile is governed by only one mode of the form $e^{-i k x}$ effectively implies that this profile is the solution of a first-order or- 
dinary differential equation. Several approaches make implicit use of this observation, ${ }^{35}$ but I have never seen this point discussed explicitly. We now illustrate this ${ }^{36}$ by deriving $v^{\dagger}$ for an equation that will turn out to be important to understanding the behavior near bifurcation points (Sec. VI),

$$
\frac{\partial \phi}{\partial t}=\frac{\partial^{2} \phi}{\partial x^{2}}+\phi+d \phi^{3}-\phi^{5} \text {. }
$$

Note that for any value of $d$, this equation is very similar to Eq. (4.1) with $b=1$ : it is invariant under a change of sign of $\phi$, and always has ${ }^{37}$ the unstable state $\phi=0$ and two absolutely stable states, $\phi= \pm \phi_{s}$, with $\phi_{s}^{2}=\left[d+\left(d^{2}\right.\right.$ $\left.+4)^{1 / 2}\right] / 2$.

Uniformly translating profiles $\phi(x-v t)$ of (4.7) must obey

$$
-v \frac{d \phi}{d x}=\frac{d^{2} \phi}{d x^{2}}+\phi+d \phi^{3}-\phi^{5} .
$$

As mentioned above, we expect the nonlinear-marginalstability profiles $\phi\left(x-v^{\dagger} t\right)$ to obey a first-order differential equation of the form

$$
\frac{d \phi}{d x}=h(\phi) \text {. }
$$

In order that solutions of this equation correspond to fronts of (4.7) that approach $\phi=0$ or $\phi= \pm \phi_{s}$ for $x \rightarrow \pm \infty, h$ needs to satisfy the constraints

$$
h(0)=0, \quad h\left( \pm \phi_{s}\right)=0 \text {. }
$$

Since solutions of (4.9) obey $d^{2} \phi / d x^{2}=h(d h / d \phi)$, the requirement that these solutions also satisfy (4.8) implies that $h$ has to satisfy

$$
-v h=h \frac{d h}{d \phi}+\phi+d \phi^{3}-\phi^{5} .
$$

The existence of two constraints (4.10) on the solutions of this first-order differential equation means that solutions will in general only exist at selected values of the velocity $v$. Indeed, upon substitution of the ansatz $h=a_{1} \phi$ $+a_{2} \phi^{2}+a_{3} \phi^{3}$ into Eq. (4.11), we find the exact solution

$$
\begin{aligned}
& h=\left(-\phi_{s}^{2} \phi+\phi^{3}\right) / \sqrt{3}, \\
& \phi(x-v t)=\left(e^{2(x-v t) \phi_{s}^{2} / \sqrt{3}}+\phi_{s}^{-2}\right)^{-1 / 2},
\end{aligned}
$$

provided $v$ and $d$ are related by

$$
v=\frac{-d+2\left(d^{2}+4\right)^{1 / 2}}{\sqrt{3}},
$$

or, equivalently,

$$
d=\frac{v \pm 2\left(v^{2}-4\right)^{1 / 2}}{\sqrt{3}} .
$$

According to (4.13) and (4.14b) the asymptotic falloff of these solutions is as $\phi \sim e^{-K x}$, with

$$
K= \begin{cases}K_{1} \equiv \frac{v-\left(v^{2}-4\right)^{1 / 2}}{2} & \text { for } d \leq \frac{2}{3} \sqrt{3} \\ K_{2} \equiv \frac{v+\left(v^{2}-4\right)^{1 / 2}}{2} & \text { for } d>\frac{2}{3} \sqrt{3}\end{cases}
$$

Since general front solutions $\phi(x-v t)$ of Eq. (4.7) fall off as $\phi \approx C_{1} e^{-K_{1} x}+C_{2} e^{-K_{2} x}$ with $K_{2}>K_{1}$, we see that the above solution satisfies the nonlinear-marginal-stability condition (3.2), $\quad C_{1}\left(v^{\dagger}\right)=0$, for $d>\frac{2}{3} \sqrt{3}$, while for $d<\frac{2}{3} \sqrt{3}$ it is just a special, but essentially uninteresting, exact solution. Therefore, for localized initial conditions, ${ }^{38}$ the asymptotic front speed of Eq. (4.7) is

$$
v^{*}=2 \text { for } d \leq d_{c}=\frac{2}{3} \sqrt{3}
$$

[as follows from applying (2.1) to (4.7)], and

$$
v^{\dagger}=\frac{-d+2\left(d^{2}+4\right)^{1 / 2}}{\sqrt{3}} \text { for } d>d_{c}=\frac{2}{3} \sqrt{3}
$$

These results are summarized in Fig. 6. We note that for positive $d$, the effect of the term $d \phi^{3}$ in (4.7) is to enhance the local growth rate over that given by the terms linear in $\phi$. As we discussed before, it is therefore not surprising that we find a transition to nonlinear marginal stability for $d$ sufficiently large and positive.

The generalization of Eq. (4.11) to Eq. (3.3) reads

$$
-v h=h \frac{d h}{d \phi}+F(\phi) \text {. }
$$

I have not been able to obtain the general solution of this equation; as a result, I can not obtain $v^{\dagger}$ for arbitrary functions $F(\phi)$. As the above analysis illustrates, however, a simple ansatz leads to an exact solution in a number of important cases.

Since an equation of the form (4.7) (generalized to a complex field) often emerges as the amplitude equation $^{39,40}$ for pattern-forming systems close to the instability threshold, it is not surprising that nonlinear marginal stability is also found in these systems. Before studying this connection, I will first present my numerical results.

\section{NUMERICAL RESULTS}

To check the validity of our picture for more complicated systems, I have studied front propagation numerically in three model equations. The first one is a straightforward extension of Eq. (4.1),

$$
\frac{\partial \phi}{\partial t}=\frac{\partial^{2} \phi}{\partial x^{2}}-\gamma \frac{\partial^{4} \phi}{\partial x^{4}}+\frac{\phi}{b}(b+\phi)(1-\phi) .
$$

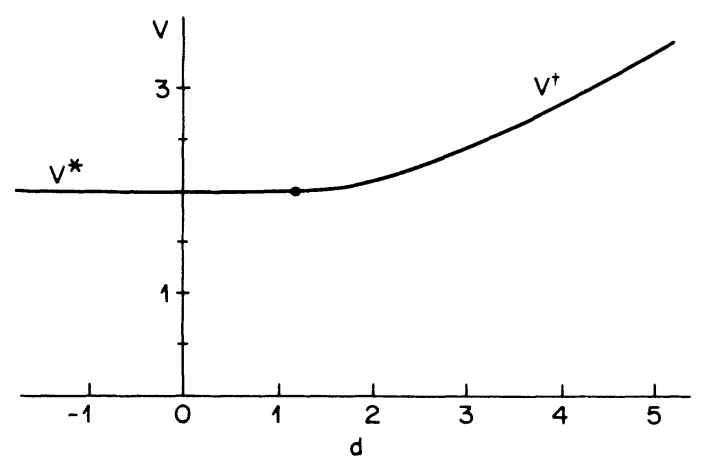

FIG. 6. Selected front speeds for Eq. (4.7). The transition to nonlinear marginal stability occurs at $d=\frac{2}{3} \sqrt{3}$. 
Since both the second- and fourth-order terms are stabilizing, the behavior of this equation for small $\gamma$ is essentially similar to that of Eq. (4.1). However, as discussed in Ref. 10, the equation exhibits a dynamical transition at $\gamma=\frac{1}{12}$ in the symmetric case $b=1$, so that for $\gamma=\frac{1}{12}$ fronts propagating into the unstable state $\phi=0$ generate a periodic array of kinks and antikinks. Although nonlinear marginal stability also occurs in the regime $\gamma>\frac{1}{12}$, I will for simplicity only report results typical for the case $\gamma<\frac{1}{12}$.

For the uniformly translating profiles $\phi_{u}(x-v t)$ relevant for Eq. (5.1) with $\gamma<\frac{1}{12}$, one can predict the nonlinear-marginal-stability value $v^{\dagger}(b)$ by solving the ordinary differential equation for $\phi_{u}$,

$-v \frac{d \phi_{u}}{d x}=\frac{d^{2} \phi_{u}}{d x^{2}}-\gamma \frac{d^{4} \phi_{u}}{d x^{4}}+\frac{\phi_{u}}{b}\left(b+\phi_{u}\right)\left(1-\phi_{u}\right)$,

and requiring that the coefficient $C_{1}$ in the large $x$ behavior $\phi_{u} \simeq \Sigma_{j} C_{j} e^{i k_{j} x}\left(\operatorname{Im} k_{1}<\operatorname{Im} k_{2}<\cdots\right)$ vanish [cf. Eq. (3.2)]. For $\gamma<\frac{1}{12}$, one finds that there is indeed a transition to nonlinear marginal stability at a value of $b$ close to $\frac{1}{2}$, the critical value for the case $\gamma=0$ discussed before (for simplicity, I only consider $0<b \leq 1$ ). The predictions for $v^{\dagger}$ for $\gamma=0.08$ resulting from this procedure are indicated in Fig. 7 by triangles.

I have also studied the actual front velocity by numerically solving the full time-dependent equation (5.1) for

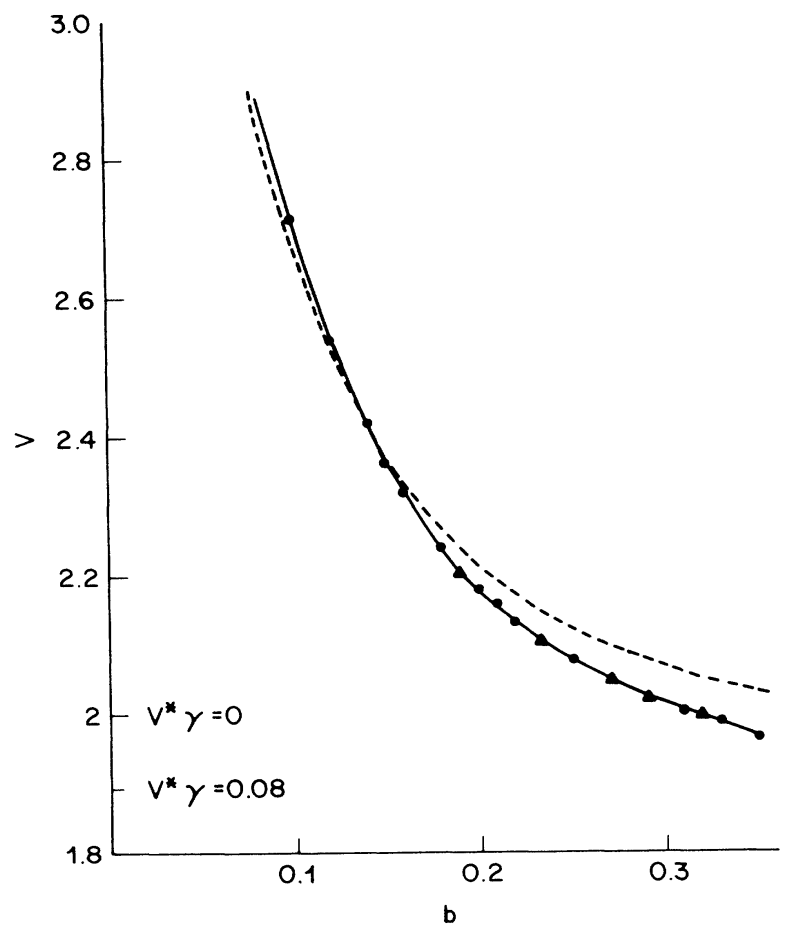

FIG. 7. Predicted and observed values of the velocity of fronts in Eq. (5.1) with $\gamma=0.08$. The triangles indicate the predicted values based on solving Eq. (5.2), as described in the text. The dots are the velocities observed in numerical solutions of Eq. (5.1). The dotted line is the analytic result for $v^{\dagger}$ at $\gamma=0$, Eq. (4.4). The values of $v^{*}$ for $\gamma=0$ and $\gamma=0.08$ are indicated on the left. various values of $b$ and $\gamma$, using an adaptation of Dee's program $^{5}$ that employs a semi-implicit finite-difference algorithm. In most of these simulations, the initial conditions were taken to be $\phi(x, t=0)=0.1 e^{-x^{2}}$, and the boundary conditions used were $\phi_{x}=\phi_{x x x}=0$ at $x=0$ and $x=\mathcal{L}$.

The measured values of the front velocity for $\gamma=0.08$ are plotted as dots in Fig. 7. As one can see, there is good agreement between the predicted values of $v^{\dagger}$ and the observed values of the front velocity.

Further evidence for the correctness of the mechanism underlying nonlinear marginal stability is shown in Fig. 8 , where I draw the leading-edge profile observed in a numerical solution of Eq. (5.1) with $\gamma=0.08$ and $b=0.1$. At the resulting velocity of $v^{\dagger}=2.715$, a linear analysis identifies three modes $e^{i k x}$ that decay for $x \rightarrow \infty$ $(\operatorname{Im} k>0)$, an imaginary one $k_{1}=0.438 i$ and a pair $k_{2}=-k_{3}^{*}=1.45+2.05 i$. In Fig. 8 , the solid line is a fit of the last two modes to the first two data points on the left. Clearly, the fit is excellent over the whole interval, showing that the slow mode $e^{i k_{1} x}$ (shown with a dashed line) is indeed absent in the leading edge. This again confirms that we have properly identified the mechanism for nonlinear marginal stability.

Figure 8 also illustrates that the stable uniformly moving profiles $\phi_{u}\left(x-v^{\dagger} t\right)$ can show oscillations about $\phi=0$, in contrast to the profiles for $\gamma=0$. The latter are always unstable if they do not fall off monotonically, ${ }^{3}$ i.e., overshoot the state $\phi=0$.

I now turn to a discussion of the other two model equations that I studied numerically, and which exhibit, to my knowledge, the first examples of nonlinear marginal stability in a pattern-forming system. In view of the above results and the general arguments presented before, I first investigate the model equation

$$
\frac{\partial \phi}{\partial t}=-2 \frac{\partial^{2} \phi}{\partial x^{2}}-\frac{\partial^{4} \phi}{\partial x^{4}}+(\epsilon-1) \phi+b \phi^{2}-\phi^{3} .
$$

For $b=0$, this equation reduces to the well-known Swift-

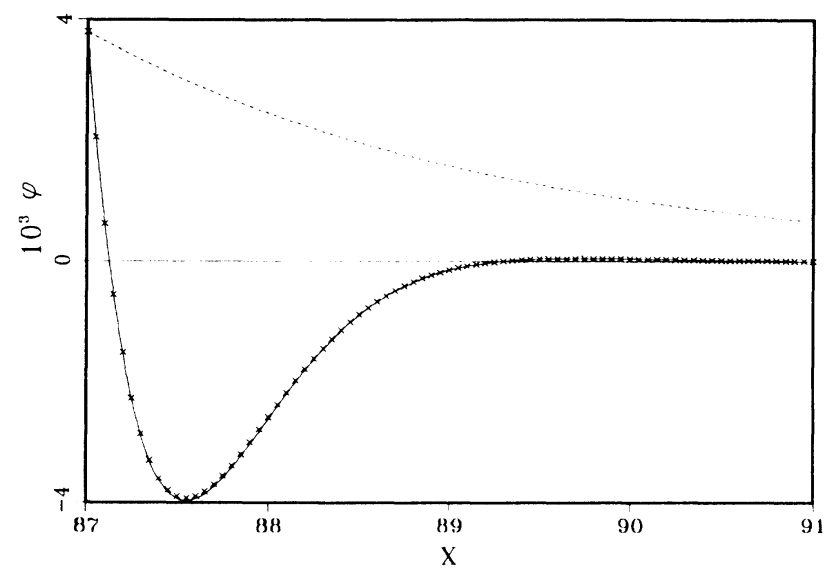

FIG. 8. Plot of the leading edge of the profile observed in Eq. (5.1) for $\gamma=0.08, b=0.1$. The observed velocity is $v^{\dagger}=2.715$. The crosses denote the values of the profile on the grid points in the simulation, while the solid line is a fit to the two modes $k_{2}$ and $k_{3}$ (see text). The dashed line illustrates the slowest mode $e^{i k_{1} x}$. 
Hohenberg equation, ${ }^{39}$ which plays an important role in understanding Rayleigh-Bénard convection and other instabilities. ${ }^{40}$ For $\epsilon<0$, the state $\phi=0$ is unstable for a band of wave vector around 1. As a result, for $\epsilon>0$ the equation admits a family of steady states that are periodic with a wavelength of about $2 \pi$. The amplitude equation describing Eq. (5.3) in the limit $\epsilon \rightarrow 0$ will be discussed in Sec. VI.

It is well known from numerical ${ }^{2,3}$ as well as rigorous analytical studies ${ }^{6}$ that front propagation into the unstable state $\phi=0$ of the Swift-Hohenberg equation $(b=0)$ is governed by linear marginal stability. According to (2.1), the velocity $v^{*}$ and wave number $k^{*}$ are in this regime given by

$$
\begin{aligned}
& v^{*}=\frac{4}{3 \sqrt{3}}(2+\sqrt{1+6 \epsilon})(-1+\sqrt{1+6 \epsilon})^{1 / 2}, \\
& \left(k^{r}\right)^{*}=\frac{1}{2}(3+\sqrt{1+6 \epsilon})^{1 / 2}, \\
& \left(k^{i}\right)^{*}=\frac{1}{2 \sqrt{3}}(-1+\sqrt{1+6 \epsilon})^{1 / 2} .
\end{aligned}
$$

Since it is observed ${ }^{2,3}$ that nodes where $\phi=0$ never disappear once they are created, one can calculate the wavelength $\lambda$ of the pattern generated by the front from the conservation-of-nodes ${ }^{2,3}$ condition $2 \pi / \lambda^{*}=\omega^{r *} / v^{*}$ $-\left(k^{r *}\right)$. This yields in the linear-marginal-stability regime

$$
\frac{2 \pi}{\lambda^{*}}=\frac{3(3+\sqrt{1+6 \epsilon})^{3 / 2}}{8(2+\sqrt{1+6 \epsilon})} .
$$

I have investigated the equation for $b>0$, and found indeed a transition to nonlinear marginal stability at some nonzero value of $b$. A possible transition is most easily located by monitoring the local value $k$ in the leading edge of the profile, since in the linear-marginalstability regime this local value is constant and equal to $k^{*}$ given by (5.5), while immediately above the transition, $k$ will be a linearly increasing function of $b$. I have determined the local value of $k$ by first locating the nodes in the leading edge to get $\operatorname{Re} k ; \operatorname{Im} k$ can then be obtained from the function values at the midpoints between the nodes. Figure 9 shows my results for $\operatorname{Im} k$ at $\epsilon=\frac{1}{4}$; the transition from linear to nonlinear marginal stability at a value $b_{c} \simeq 1.52 \pm 0.08$ is immediately obvious. The error bars in this figure indicate the variation in $k$ in that part of the leading edge where the extrema in $\phi$ range between 0.002 and 0.0002 in absolute value. Note that in the linear-marginal-stability regime $(b \lesssim 1.5)$ the measured values of $k^{i}$ appear to be below $k^{i *}$. This is due to the importance of corrections to the exponential behavior in this regime. Indeed, at the linear-marginal-stability point two roots coincide, so that $\phi$ is asymptotically of the form $\phi \sim C_{1} e^{i k^{*} x}+C_{2} x e^{i k^{*} x}$, which implies that the locally measured value of $k$ behaves as $k^{*}-i / x$ (see Sec. VIII for further details). In the nonlinear-marginal-stability regime, on the other hand, the front profile is purely exponential for large $x$, and the estimated error in $k$ is of the order of the size of the dots. In Fig. 10, I plot the leading-edge values of $\operatorname{Im} k$ and the velocity $v$ observed on the curve $v\left(k^{i}\right)$ defined by the requirement $\partial \omega^{i} / \partial k^{r}=0$ (cf. Fig. 2). Again, we see how in the nonlinear-

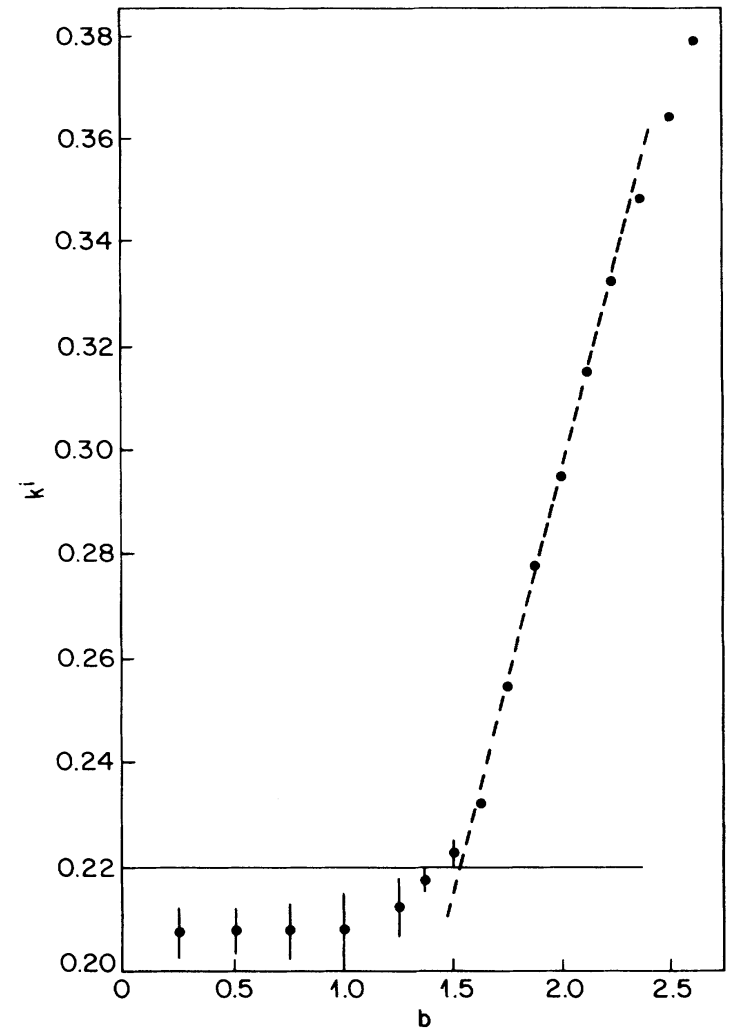

FIG. 9. The measured value of $k^{i}$ in the leading edge where the maxima of $\phi$ are between 0.0002 and 0.002 in absolute value, for solutions of Eq. (5.3) with $\epsilon=\frac{1}{4}$. The solid line indicates the linear-marginal-stability value. See text for an explanation why the error bars for $b \lesssim 1.5$ are large. Results are based on numerical solutions with 19600 grid points at a spacing of 0.03 . The time step was 0.01 and measurements were made at time $t=205$.

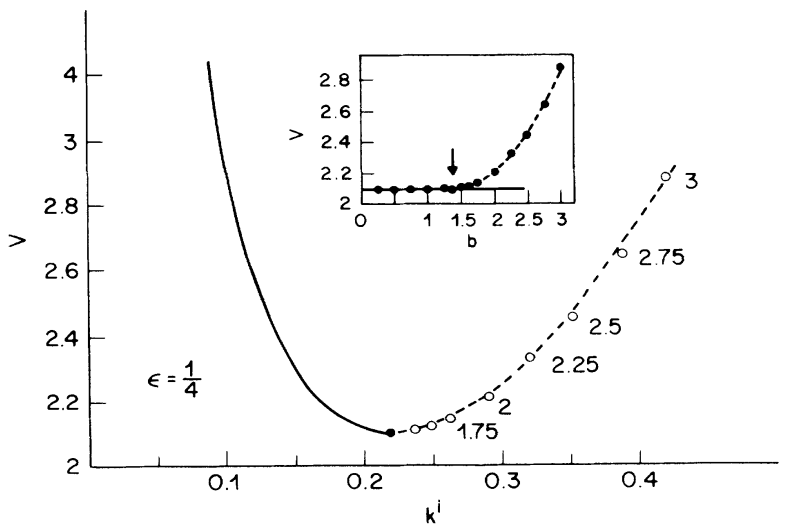

FIG. 10. $v$ as a function of the observed values of $k^{i}$ in the leading edge, for Eq. (5.3) with $\epsilon=\frac{1}{4}$. The solid dot marks the marginal-stability value, and the open dots the observed velocities and values of $k^{i}$ at the indicated value of $b$. The curve is a plot of the function $v\left(k^{i}\right)$ obtained by solving $\partial \omega^{i} / \partial k^{r}=0$. The inset depicts the observed velocities as a function of $b$. 
marginal-stability regime $b>b_{c}$ the profile in the leading edge is determined by the second smallest root of $v\left(k^{i}\right)$. The resulting front velocity as a function of $b$ is also drawn in the inset; since the curve $v\left(k^{i}\right)$ has a minimum at $v^{*}$, the linear increase in $k^{i}$ above $b_{c}$ (Fig. 9) corresponds to a quadratic dependence in $v$. Indeed, upon expanding the expression $v=\omega^{r} / k^{r}$ about the marginalstability point value given by Eq. (2.1), one gets

$$
v=v^{*}+\frac{1}{2 k^{r *}} \operatorname{Re}\left[\left.\frac{d^{2} \omega}{d k^{2}}\right|_{k^{*}}(\Delta k)^{2}\right)+\cdots,
$$

where $\Delta k^{r}$ and $\Delta k^{i}$ are to lowest order related by

$$
\left.\operatorname{Im} \frac{d^{2} \omega}{d k^{2}}\right|_{k^{*}} \Delta k^{r}+\left.\operatorname{Re} \frac{d^{2} \omega}{d k^{2}}\right|_{k^{*}} \Delta k^{i}=0 .
$$

The latter result is obtained by expanding the condition $\partial \omega^{i} / \partial k^{r}=\operatorname{Im}(d \omega / d k)=0$ to first order in $\Delta k$. The velocity data plotted in the inset of Fig. 10 are in good agreement with Eqs. (5.7) and (5.8).

Two snapshots of the front profiles for $b=1.625$ and 2.75 are shown in Fig. 11; note how the nonlinear part of
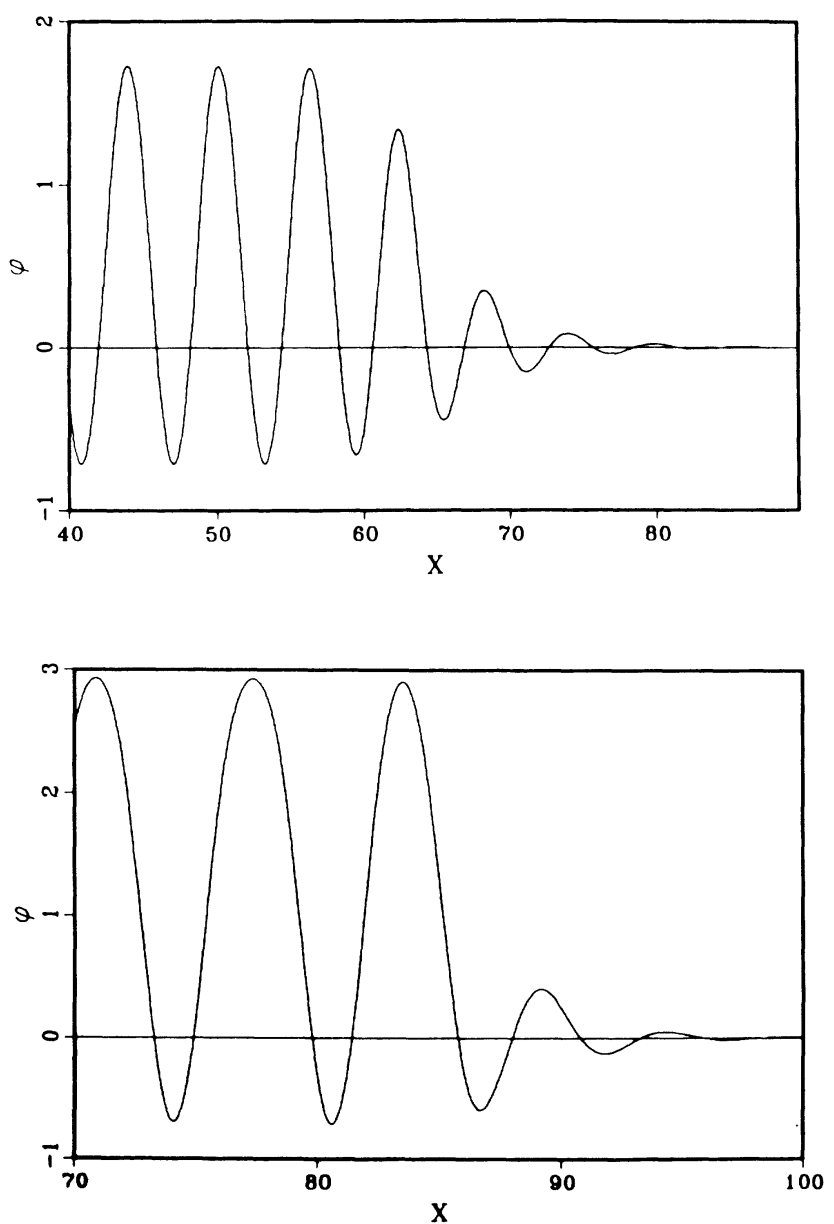

FIG. 11. Top: Snapshot of the front profile in the extension of the Swift-Hohenberg equation, Eq. (5.3), for $b=1.625$ and $\epsilon=\frac{1}{4}$. As Figs. 9 and 10 show, this value is just above the transition to nonlinear marginal stability. Bottom: same for $b=2.75$. Note the sharpness of the front at this value of $b$. the front tends to sharpen for increasing values of $b$.

I have also studied the analogue for the SwiftHohenberg equation of Eq. (4.7),

$$
\frac{\partial \phi}{\partial t}=-2 \frac{\partial^{2} \phi}{\partial x^{2}}-\frac{\partial^{4} \phi}{\partial x^{4}}+(\epsilon-1)+d \phi^{3}-\phi^{5} .
$$

The measured values of $k^{i}$ as a function of $d$ are shown in Fig. 12 for $\epsilon=\frac{1}{4}$. Qualitatively, the results resemble those of Fig. 9; the transition to nonlinear marginal stability occurs in this case at $d=0.63 \pm 0.05$. Besides $v$, also the wavelength $\lambda$ of the pattern generated by the front will differ for $d>d_{c}$ from the value $\lambda^{*}$ given by Eq. (5.6). However, for systems for which the conservation-ofnodes condition $2 \pi / \lambda=\omega^{r} / v-k^{r}$ holds, one obtains, upon expanding the right-hand side around the marginal stability point given by Eq. (2.1),

$$
\begin{aligned}
\frac{2 \pi}{\lambda}-\frac{2 \pi}{\lambda^{*}} & =\left.\frac{1}{2 v^{*}} \operatorname{Re} \frac{d^{2} \omega}{d k^{2}}\right|_{k^{*}}\left(\Delta k^{r}\right)^{2}-\frac{\omega^{r^{*}}}{v^{* 2}} \Delta v+\cdots \\
& =O(\Delta k)^{3}
\end{aligned}
$$

where we used Eq. (5.7) for $\Delta v$. Thus, due to the cancellation of the second-order terms, $\lambda$ does not vary quadratically, but as $(\Delta k)^{3}$ near the transition (a similar cancellation of terms is found at the transition from uniformly translating fronts to envelope fronts studied in Ref. 10). In pattern-forming systems, it will therefore be difficult to discern the transition from linear to nonlinear marginal stability from the wavelength of the emerging pattern. Figure 13 shows the data for $v$ and $\lambda$ for Eq. (5.9) with $\epsilon=\frac{1}{4}$. As before, the quadratic increase of $v$ with $d$ is borne out by our simulations. In agreement with (5.10), the relative change in $\lambda$ over the parameter range investigated is indeed very small: while $k^{i}$ and $v^{\dagger}$ change by about $60 \%$ and $20 \%$, respectively, $\lambda$ varies by

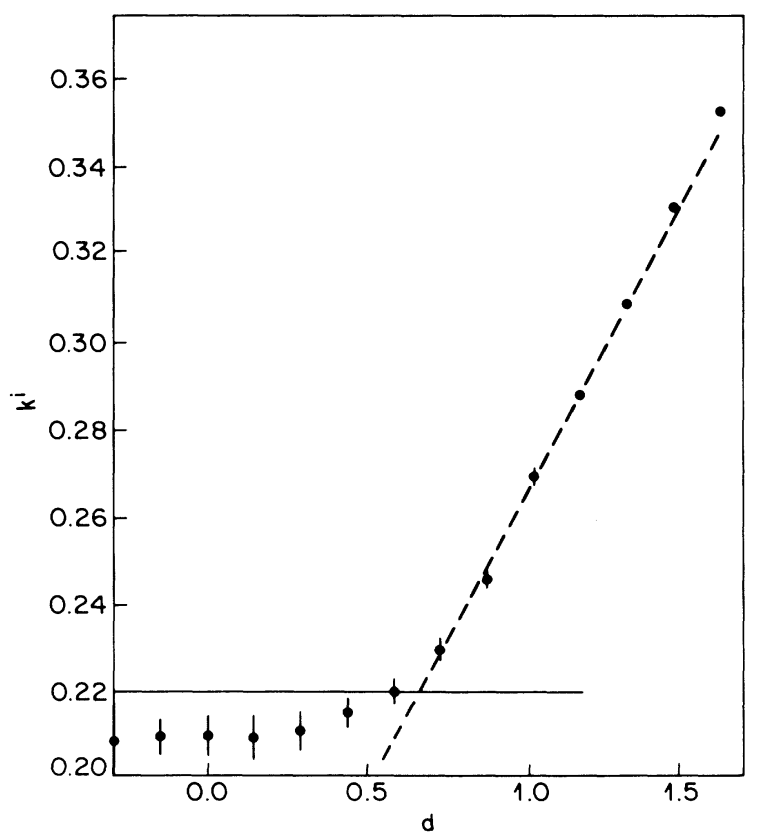

FIG. 12. As Fig. 9, but for Eq. (5.9) instead of Eq. (5.3). 

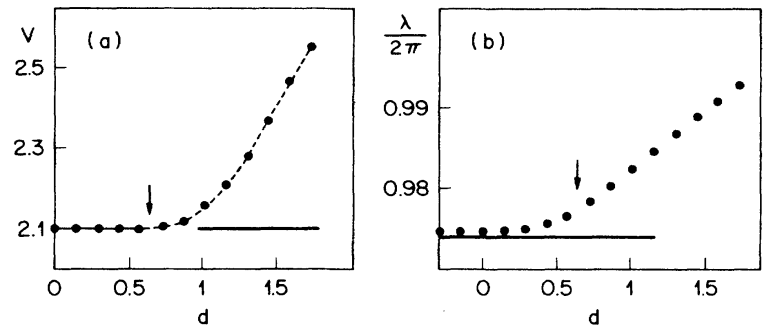

FIG. 13. (a) Measured front velocity for Eq. (5.9) with $\epsilon=\frac{1}{4}$. The horizontal line denotes the linear-marginal-stability value. The arrow indicates the transition from linear to nonlinear marginal stability. (b) The wavelength $\lambda$ of the pattern generated behind the front for Eq. (5.9) with $\epsilon=\frac{1}{4}$. These measurements are made after a time of 205 , at which point the wavelength was still found to be decreasing slightly, especially near $d \simeq 0.6$. Note the scale of the graph: the deviation from the expected behavior is less than $0.4 \%$.

only $2 \%$ in the parameter range we investigated. In fact, with such small variations, my numerical simulations are not accurate enough to test the cubic variation of $\lambda$ with $\Delta k$, as the pattern wavelength was still slowly changing when the measurements were made at time 205 .

The numerical results presented in this section provide convincing evidence for the correctness of our picture for the nonlinear-marginal-stability mechanism. Unfortunately, especially for pattern-forming systems like (5.3) and (5.9), we see at present no way to predict the occurrence of nonlinear marginal stability analytically far away from threshold.

\section{BEHAVIOR CLOSE TO BIFURCATION POINTS}

Although our understanding of the mechanisms of front propagation into unstable states appears to be quite complete, we cannot in general predict the occurrence of nonlinear marginal stability for pattern-forming systems like (5.3) and (5.9) without performing a fully nonlinear analysis of the dynamics of the relevant equations. Fortunately, however, this type of front propagation is most relevant close to a threshold for instability: experimental systems are most easily quenched into an unstable state when one works close to the instability threshold. Moreover, in the important case of systems with slowly varying (control) parameters, global instabilities occur when the systems are locally weakly unstable in an extended region. ${ }^{28}$ Close to an instability threshold, many aspects of pattern-forming systems can be understood by studying the appropriate amplitude equation. ${ }^{39,40}$ The amplitude equation that describes the near-threshold behavior of systems with a pitchfork bifurcation ${ }^{41}$-including Eq. (5.3) and (5.9) -is a complex extension of Eq. (4.7). Let us therefore first rewrite Eq. (4.7) as

$$
\frac{\partial \phi}{\partial t}=\frac{\partial^{2} \phi}{\partial x^{2}}+\epsilon \phi+c_{1} \phi^{3}-c_{2} \phi^{5}, \quad c_{2}>0,
$$

and reformulate our results in a language more suitable for a bifurcation analysis. For positive $\epsilon$, this equation reduces, upon rescaling of $\phi, x$, and $t$, to an equation of the form (4.7) with $d=c_{1} / \sqrt{\epsilon c_{2}}$. In many applications, however, the equation arises in the above form with $\epsilon$ a small control parameter that can change sign, and $c_{1}$ and $c_{2}$ of order unity. The nature of the bifurcation at $\epsilon=0$ is then determined by the sign of $c_{1}$. For $c_{1}$ negative, the homogeneous steady state $\phi=0$ that is stable for $\epsilon<0$ bifurcates to two stable states $\phi \neq 0$ at $\epsilon=0$; this so-called supercritical (forward) bifurcation ${ }^{41}$ is depicted in Fig. 14(a). For $c_{2}>0$, the bifurcation is a subcritical ${ }^{41}$ (inverted) one: now there are three steady states $\phi=$ const in the range $-\frac{1}{4}<\epsilon c_{2} / c_{1}^{2}<0$. The two stable states for $\epsilon<0$ are drawn with a solid line in Fig. 14(b). To depict the unstable states, we have used the convention that they are drawn with a dashed line if fronts propagating into these states are governed by nonlinear marginal stability, while a dotted line is used when the fronts are governed by linear marginal stability. ${ }^{42}$ For $\epsilon>0$, the results shown in Fig. 14 follow directly from those derived earlier for $\mathrm{Eq}$. (4.7): for the supercritical bifurcation $\left(c_{1}<0\right)$, fronts propagating into the unstable state $\phi=0$ are always governed by linear marginal stability (provided the initial conditions are sufficiently localized), and $v^{*}=2 \sqrt{\epsilon}$. If the bifurcation is subcritical, however, nonlinear marginal stability always governs front propagation into the unstable state $\phi=0$ sufficiently close to threshold. The transition to marginal stability occurs at a value $\epsilon c_{2} / c_{1}^{2}=(\sqrt{3} / 2)^{2}=\frac{3}{4}$. Thus the transition towards linear marginal stability will occur toward smaller $\epsilon$ for decreasing $c_{1}$, i.e., when the bifurcation becomes more weakly first order.

Regarding the stability of the stable states for $\epsilon<0$, we note that with the aid of the fact that this equation can be derived from a Lyapunov functional

$$
F=\int d x\left(\frac{\partial \phi}{\partial x}\right)^{2}-\frac{1}{2} \epsilon \phi^{2}-\frac{1}{4} c_{1} \phi^{4}+\frac{1}{6} c_{2} \phi^{6},
$$

it is easy to show that the state $\phi=0$ is the absolutely

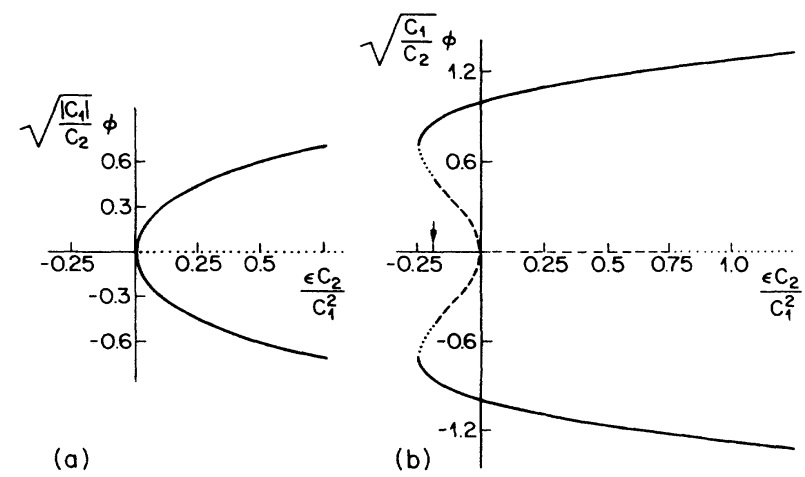

FIG. 14. Bifurcation diagram of the steady states $\phi=$ const of Eq. (6.1). Solid lines denote stable states and dotted (dashed) lines unstable states whose front propagation is governed by linear (nonlinear) marginal stability. (a) Supercritical bifurcation for $c_{1}<0$. (b) Subcritical bifurcation for $c_{1}>0$. To the left of the arrow, the steady state $\phi=0$ is the absolutely stable state, to the right of it this state becomes metastable. 
stable state for $\epsilon c_{2} / c_{1}^{2}<-\frac{3}{16}$; for larger $\epsilon$, the other stable state with $\phi \neq 0$ is the absolutely stable one. For $\epsilon<0$, the analysis of Sec. IV still yields an exact front solution, and since the interfacial profile between two stable states is unique, the velocity is given by the same formula. Taking into account the different scaling of space and time in Eqs. (4.7) and (6.1), one finds for the velocity of the front between $\phi=0$ and the stable state $\phi \neq 0$,

$$
\begin{aligned}
& v^{\dagger}=\frac{c_{1}}{\sqrt{3 c_{2}}}\left[-1+2\left(1+4 \epsilon c_{2} / c_{1}^{2}\right)^{1 / 2}\right] \\
& c_{1}>0,-\frac{1}{4}<\frac{\epsilon c_{2}}{c_{1}^{2}}<\frac{3}{4} .
\end{aligned}
$$

The fact that this velocity is negative for $\epsilon c_{2} / c_{1}^{2}<-\frac{3}{16}$ expresses that in this range the domain where $\phi=0$ grows rather than shrinks, since $\phi=0$ is the absolutely stable state. ${ }^{43}$ The profiles $\phi\left(x-v^{\dagger} t\right)$ can be obtained from Eq. (4.13).

Although these predictions for the subcritical bifurcation will be quantitatively correct only for weakly subcritical bifurcations $\left(c_{1}\right.$ small) that are accurately described by Eq. (6.1), I expect this picture to be qualitatively correct in general. For a supercritical bifurcation, the lowest-order nonlinearity reduces the growth rate away from the unstable state, and the analysis of Sec. IV shows that linear marginal stability will be operative for sufficiently small $\epsilon$, with $v^{*} \sim \sqrt{\epsilon}$. For a subcritical bifurcation, a front velocity $v \sim \sqrt{\epsilon}$ must be incorrect for small $\epsilon$, since the finite driving force from the nonlinear terms will yield a finite front velocity $v^{\dagger}$ for $\epsilon \rightarrow 0$. This effect is stronger, the more first order the transition is.

These results bear immediately on a large class of pattern-forming systems that exhibit a stationary bifurcation whose near-threshold behavior is governed by the complex amplitude equation ${ }^{44}$

$$
\begin{gathered}
\frac{\partial \Phi}{\partial t}=\frac{\partial^{2} \Phi}{\partial x^{2}}+\epsilon \Phi+c_{1}|\Phi|^{2} \Phi-c_{2}|\Phi|^{4} \Phi \\
-i\left(k_{1} \epsilon \frac{\partial \Phi}{\partial x}+k_{2} \frac{\partial^{3} \Phi}{\partial x^{3}}-\left(k_{3}+k_{4}\right)|\Phi|^{2} \frac{\partial \Phi}{\partial x}\right. \\
\left.-\left(k_{3}+k_{5}\right) \Phi^{2} \frac{\partial \Phi^{*}}{\partial x}\right)+\cdots
\end{gathered}
$$

Note that terms even in the complex amplitude $\Phi$ are generally absent for translationally invariant systems, as a translation corresponds to a multiplication of $\Phi$ by a phase factor $e^{i \zeta}$. The coefficients $k_{1}$ through $k_{5}$ are chosen so as to conform to the notation by Cross et al. ${ }^{45}$ When for a particular system $c_{1}<0$, earlier work ${ }^{3}$ as well as the above arguments show that front propagation is governed by linear marginal stability for small enough $\epsilon$. From the above discussion, we anticipate that the transition will occur for $c_{1}$ of order $\epsilon^{1 / 2}$ (provided $c_{2}>0$ ). Since spatial variation in $\Phi$ is on the scale $\epsilon^{-1 / 2}$, we see that in this regime, where $c_{1}=O\left(\epsilon^{1 / 2}\right)$, the terms $|\Phi|^{2} \partial \Phi / \partial x$ and $\Phi^{2} \partial \Phi^{*} / \partial x$ are of the same order as the other terms, viz., of order $\epsilon^{5 / 4}$. Hence, whenever one of the coefficients $k_{3}, k_{4}$, and $k_{5}$ is nonzero, Eq. (6.4) does not reduce to Eq. (6.1) in lowest order, and we can only conclude that the transition occurs at a critical value $c_{1, c}(\epsilon)=O\left(\epsilon^{1 / 2}\right)$. For $k_{3}=k_{4}=k_{5}=0$ and $c_{2}>0$, on the other hand, Eq. (6.4) is, in lowest order, nothing but the extension of (6.1) to complex $\phi$; one can convince oneself that the transition in the complex equation occurs at the same parameter values as in the real equation (6.1). We thus get

$$
c_{1, c}=\sqrt{(4 / 3) \epsilon c_{2}}+\cdots, \quad k_{3}=k_{4}=k_{5}=0 .
$$

Let us apply these results to the two extensions of the Swift-Hohenberg equation that we introduced. In Appendix D, I show that for Eq. (5.3) one has

$$
c_{1}=\frac{38}{27} b^{2}-1, \quad k_{4}=\frac{16 b^{2}}{81}, \quad k_{1}=k_{3}=k_{5}=0
$$

and for Eq. (5.9)

$$
c_{1}=d, \quad c_{2}=\frac{10}{9}, \quad k_{1}=k_{3}=k_{4}=k_{5}=0 .
$$

Thus the above results imply that the transition to nonlinear marginal stability occurs at

$$
b_{c}(\epsilon)=\sqrt{27 / 38}+O\left(\epsilon^{1 / 2}\right),
$$

when Eq. (5.3) is used, and

$$
d_{c}(\epsilon)=\sqrt{(40 / 27) \epsilon}+\cdots,
$$

when Eq. (5.9) is used. In Fig. 15, I compare these pre-

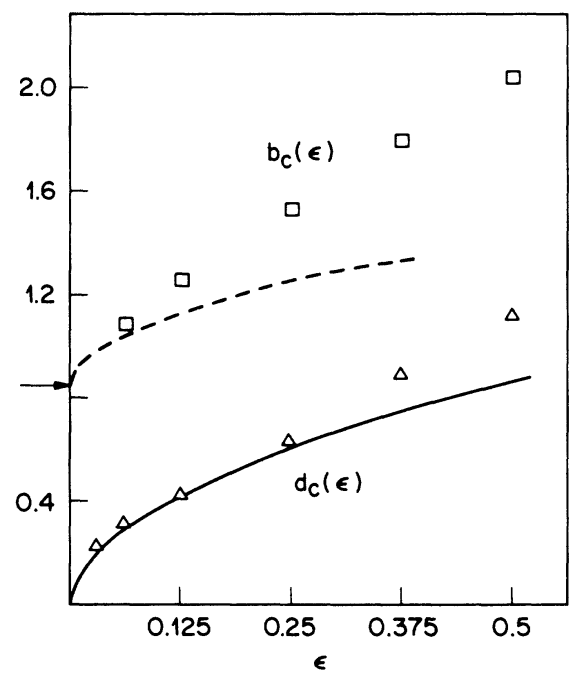

FIG. 15. Values of the dimensionless quantities $b_{c}(\epsilon)$ (squares) and $d_{c}(\epsilon)$ (triangles) at which the transition from linear to nonlinear marginal stability occurs in Eqs. (5.3) and (5.9), as obtained from the numerical simulations. The uncertainty in these values is of the order of the size of the symbols. The arrow indicates our prediction for $b_{c}(\epsilon=0)$, the solid line our prediction (6.9) for $d_{c}(\epsilon)$ to lowest order in $\epsilon$, and the dashed line the approximation (D23) for $b_{c}(\epsilon)$. Each numerical data point was determined on the basis of a plot such as Fig. 9 for numerical solutions at time 205 with 19600 grid points, a spacing of 0.03 , and a time step of 0.01 . 
dictions with my numerical results for a number of values of $\epsilon$. Clearly, the lowest-order prediction for $d_{c}$, shown with a solid line, agrees to within the numerical error with the numerical data for $\epsilon \lesssim \frac{1}{4}$. The data for $b_{c}(\epsilon)$ also appear to be consistent with our result for $\epsilon=0$, which is indicated by the arrow. We also plot with a dashed line the approximation obtained by neglecting the $k_{4}|\Phi|^{2} \partial \Phi / \partial x$ term so that $c_{1, c}=\sqrt{(4 / 3) \epsilon c_{2}}$ (the coefficient $c_{2}$ is given in Appendix D). Although the coefficient $k_{4}$ is rather small, this approximation only appears to be reasonably accurate for quite small $\epsilon$.

\section{DIFFERENCE WITH EXCITABLE DYNAMICS IN CHEMICAL WAVES}

As mentioned in the Introduction, apart from the experiments by Hanna et al. ${ }^{17}$ most aspects of traveling waves in chemical systems are not associated with front propagation into linearly unstable states, but with bistability. Although these differences can be extracted from the literature, ${ }^{19,20}$ we feel it worthwhile to underscore the distinction some more.

The general structure ${ }^{18,19,46}$ that leads to excitable dynamics and chemical waves is the following model for two coupled chemical reactions:

$$
\begin{gathered}
\epsilon \frac{\partial u}{\partial t}=\epsilon^{2} D_{1} \frac{\partial^{2} u}{\partial x^{2}}+f(u, w), \\
\frac{\partial w}{\partial t}=\epsilon^{2} D_{2} \frac{\partial^{2} w}{\partial x^{2}}+g(u, w),
\end{gathered}
$$

with $\epsilon<<1$. The presence of the small coefficient in front of the time derivative of $u$ in (7.1a) implies that the dynamics of $u$ is on a much faster time scale than that of $w$. In lowest order of approximation $u$ is therefore driven rapidly (on the fast time scale) to one of the stable zeroes of $f(u, w)$ while $w$ essentially remains unchanged. Typically, it is assumed that the zeroes of $f(u, w)$ fall on a curve in the $u-w$ plane such as the one sketched in Fig. 16(a). The branches on which $A$ and $B$ lie are supposed to be stable with respect to small perturbation in $u$, so that $f(u, w=$ const $)$ is of the form sketched in Fig. 16(b).

For simplicity, we illustrate the basic ingredients that lead to traveling pulses in the simplest case where $D_{2}=0$. Suppose $w$ is initially constant. As argued above, $u$ will (a)

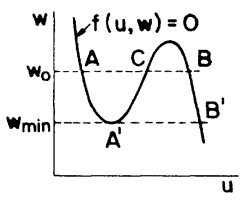

(b)

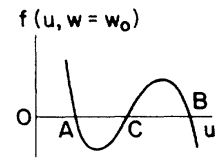

(c)

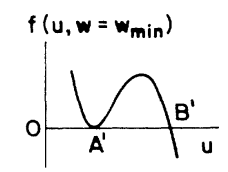

FIG. 16. (a) Schematic behavior of the function $f$ used in model (7.1) for excitable behavior. The solid line indicates the values of $u$ and $w$ where $f$ vanishes. (b) Assumed behavior of $f$ as a function of $u$ for fixed $w$. (c) When $w=w_{\min }$ as indicated in (a), two zeros of $f\left(u, w=w_{0}\right)$ merge. For $w_{0}<w_{\min }, f\left(u, w_{0}\right)$ has only one zero. rapidly be driven to one of the stable zeros of $f(u, w)$. Suppose also that $u$ is driven to point $A$ in Fig. 16(a) in one region and to point $B$ in the other region. In general, one of these states, say $A$, is metastable, while the other one $(B)$ is stable. Therefore, a frontlike wave will start to propagate into state $A$. Clearly, at this level we encounter front propagation into a metastable state due to bistability instead of front propagation into a linearly unstable state.

On the slow time scale, $w$ is changing according to Eq. (7.1b); thus the value of $w_{0}$ in Fig. 16(a) is slowly drifting in time. Consequently, points $A$ and $B$ are slowly moving up or down the curve, while the front solution between these two states adiabatically follows this time dependence. We refer to a detailed discussion of the ensuing dynamics to Refs. 18 and 19, and focus only on one particular aspect of relevance to the rest of this paper. Suppose $g$ in $(7.1 \mathrm{~b})$ is such that $w$ is decreasing in time, and follow $w$ till the value $w_{\text {min }}$ corresponding to the minimum of the left branch of zeros of $f(u, w)$-see Fig. 16(a). At this point, $f\left(u, w=w_{\text {min }}\right)$ is of the type sketched in Fig. 16(c). Since the point $A^{\prime}$ is now unstable, one can view this particular front between $A^{\prime}$ and $B^{\prime}$ as a front propagating into an unstable state. We have already encountered such an example where a stable and unstable point coalesce before in Sec. VI [Eq. (6.1) for $\left.\epsilon c_{2} / c_{1}^{2}=-\frac{1}{4}\right]$, and one can show ${ }^{47}$ that the front velocity is just continuous and smooth upon approaching this point. In practice, this particular aspect is not of much relevance, since as soon as $w$ is driven slightly below this minimum, the term $f$ on the right-hand side of $(7.1 \mathrm{a})$ becomes nonzero, and $u$ is driven rapidly to a value close to $B^{\prime} ;$ this is the dynamics most relevant to excitable media. ${ }^{18,19}$

\section{APPROACH TO THE ASYMPTOTIC VALUE}

We will now derive the rate of approach of the front velocity to the asymptotic linear-marginal-stability value $v^{*}$. Apart from work by Bramson ${ }^{32}$ on equations of the form (3.3), not much attention has been paid to this question. While our analysis applies only to the leading edge of general front propagation equations, our expression is consistent with one of the rigorous results by Bramson ${ }^{32}$ in the special case of Eq. (3.3). Our expression is different from the one usually given in the pinch-point analysis. ${ }^{22,29}$

It is useful to first note the following. For a profile moving at an arbitrary velocity, the asymptotic special decay of a profile is of the form $\phi \simeq C_{1} e^{i k_{1} x}+C_{2} e^{i k_{2} x}$ $+\cdots$. However, the linear-marginal-stability point is exactly the point where the root $k^{*}$ is degenerate (two roots coincide), and this implies ${ }^{48}$ that the spatial falloff at the velocity $v^{*}$ is $\phi \simeq C_{1} e^{i k^{*} x}+C_{2} x e^{i k^{*} x}+\cdots$. Upon writing $x e^{i k^{*} x}=e^{i k^{*} x+\ln x}$, we see that this implies that in the leading edge, the local wave number $q=\partial u / \partial x$ defined before Eq. (2.2) will vary for large $x$ as

$$
q \simeq k^{*}-\frac{i}{x}, \quad x \rightarrow \infty .
$$

In Sec. II, we wrote the local wave number $q$ as a func- 
tion of $u^{i}$ to bring out the structure of the equation for general front profiles. However, in considering the lowest-order terms in expanding (2.2) around the linearmarginal-stability value, we can return to a comoving variable $x^{\prime}=x-v^{*} t$ since to lowest order $u^{i} \simeq k^{i}{ }^{*}{ }^{\prime}$ $+O\left(\ln x^{\prime}\right)$. Furthermore, (8.1) shows that spatial derivatives $q_{x x x}$ can be neglected compared to the term proportional to $q_{x x}$ in $\mathcal{L}$ (in this and Sec. IX, the subscript $x$ will denote a partial differentiation with respect to $\left.x^{\prime}\right)$. Thus upon writing $q=k^{*}+\rho\left(x^{\prime}, t\right)$, the dominant terms from Eq. (2.2) are [cf. also Eq. (3.26) of Ref. 9]

$$
\rho_{t}=-\left.f_{q q}\right|_{q=k} * \rho \rho_{x}-\left.f_{q_{x}}\right|_{q=k} * \rho_{x x} .
$$

As explained just before Eq. (2.2), the function $f\left(q, q_{x}, \ldots\right)$ is obtained from substituting the ansatz $\phi=e^{i u(x, t)}$ into the linearized dynamical equation. This implies, however, that there are various relations between the derivatives $\left.f_{q_{x}}\right|_{k},\left.f_{q q}\right|_{k}$, etc.-in fact all information about the linearized equation is contained in the dispersion relation $\omega(k)$, and in Sec. IX, we will indeed show that [cf. Eq. (9.4)]

$$
\left.i f_{q_{x}}\right|_{q=k^{*}}=\left.\frac{1}{2} f_{q q}\right|_{q=k^{*}}=\left.\frac{1}{2} \frac{d^{2} \omega}{d k_{2}}\right|_{k^{*}} .
$$

We can therefore write $(6.2)$ as

$$
\rho_{t}=-\left.\frac{d^{2} \omega}{d k^{2}}\right|_{k}\left(\rho \rho_{x}-\frac{i}{2} \rho_{x x}\right) \text {. }
$$

Equation (8.1) shows that the relevant-time-independent solution should be $\rho=-i / x^{\prime}$, and indeed it is easy to verify that this is a steady-state solution of Eq. (8.4).

Equation (8.3) is a complex version of the well-known Burgers equation. ${ }^{49}$ As in the Burgers equation we expect that the long time decay of $\rho(x, t)$ towards the solution $\rho=-i / x^{\prime}$ will be governed by a self-similar solution of the form

$$
\rho(x, t)=\frac{1}{x^{\prime}} g\left(\frac{x^{\prime 2}}{t}\right), \quad g(0)=-i
$$

The unique function $g$ that satisfies the boundary condition $g(0)=-i$ turns out to be the following exact solution of (8.4):

$$
\rho(x, t)=-\frac{i}{x^{\prime}}+\left(\left.\frac{d^{2} \omega}{d k^{2}}\right|_{k^{*}}\right)^{-1} \frac{x^{\prime}}{t} .
$$

Of course, this solution is only physically relevant when the second term is small compared to the first, i.e., for $x^{\prime 2} / t<<1$ (remember that $x^{\prime}$ is the coordinate in a frame moving with velocity $v^{*}$ ). We can now obtain the envelope velocity in this comoving frame, defined by $v=-\operatorname{Im} u_{t} / \operatorname{Im} q=\operatorname{Im} f\left(q, q_{x}, \ldots\right) / \operatorname{Im} q$. Upon expanding about the marginal stability point (2.1), we get with $f(q=k)=\omega(k),(8.3)$ and (8.6) to lowest order

$$
\begin{aligned}
v & =\frac{1}{\left(k^{i}\right)^{*}} \operatorname{Im}\left(\frac{1}{2} f_{q q}^{*} \rho^{2}+f_{q_{x}}^{*} \rho_{x}\right) \\
& =\frac{1}{\left(k^{i}\right)^{*}} \operatorname{Im}\left[\left.\frac{1}{2} \frac{d^{2} \omega}{d k^{2}}\right|_{k^{*}}\left(\rho^{2}-i \rho_{x}\right)\right] \\
& =-\frac{3}{2\left(k^{i}\right)^{*} t}\left[1+O\left[\frac{x^{\prime 2}}{t}\right]\right] .
\end{aligned}
$$

Since this is the velocity in a frame moving with speed $v^{*}$, the final result for the front velocity is

$$
v=v^{*}-\frac{3}{2\left(k^{i}\right)^{*} t} .
$$

It is interesting that this expression only depends implicitly on the dispersion relation $\omega(k)$ through $\left(k^{i}\right)^{*}$. Note also that $v$ approaches $v^{*}$ from below due to the partial cancellation of the first and second term on the righthand side of (8.2). Thus the naive statement of Sec. II that the velocity gradually slows down to $v^{*}$ is incorrect for long times.

A few comments are in order. First of all, although our derivation started from Eq. (2.2) which is only valid when the dynamical equations involve first-order time derivatives only, one can easily convince oneself that higher-order time derivatives would not affect the similarity solution (8.5). Hence the results (8.8) should hold for more general equations as well.

Furthermore, Eq. (8.8) has been derived in the leading edge only, and it is not a priori clear why the rate of convergence of the velocity should be the same everywhere on the profile. Nevertheless, Bramson ${ }^{32}$ has proved precisely this for uniformly translating profiles of Eq. (3.3), governed by linear marginal stability. In other words, he has shown that with localized initial conditions, $\phi(x+v(t), t)$ converges to a steady-state profile $\phi_{s}(x)$ uniformly in $x$. For $v(t)$, he finds $v(t)=2-3 / 2 t+\cdots$. Since $\left(k^{i}\right)^{*}=1$ for equations of the form (3.3), our result (6.8) is consistent with Bramsom's rigorous result. Our expression and the result of Bramson ${ }^{32}$ for (3.3) are in disagreement with the expression for the long-time asymptotic of the profile $\phi$ often quoted in the pinchpoint analysis, ${ }^{22,29}$ and a similar expression obtained by Kamenskii and Manakov, ${ }^{7}$

$$
\phi \sim t^{-1 / 2} e^{i k^{*} x-i \omega^{*} t},
$$

which implies

$$
v=v^{*}-\frac{1}{2\left(k^{i}\right)^{*} t} .
$$

We believe that this is due to the fact that, in spite of claims to the contrary, ${ }^{22} \mathrm{Eq}$. (8.9) is not valid uniformly in $x$. Indeed, Eq. (8.9) is also inconsistent with the spatial dependence one expects because two roots coincide, $\phi \sim x e^{i k}{ }^{*}$. In Appendix $\mathrm{C}$, we discuss this in more detail, and show how Eqs. (8.6) and (8.8) appear to emerge from a more careful analysis in the pinch-point approach.

I have tested the above predictions through numerical simulations. In Fig. 17, I plot $v$ versus $t^{-1}$ for Eq. (4.1) with $b=1$. The numerical results clearly favor the expression derived by Bramson ${ }^{32}$ and me over Eq. (8.10). In 


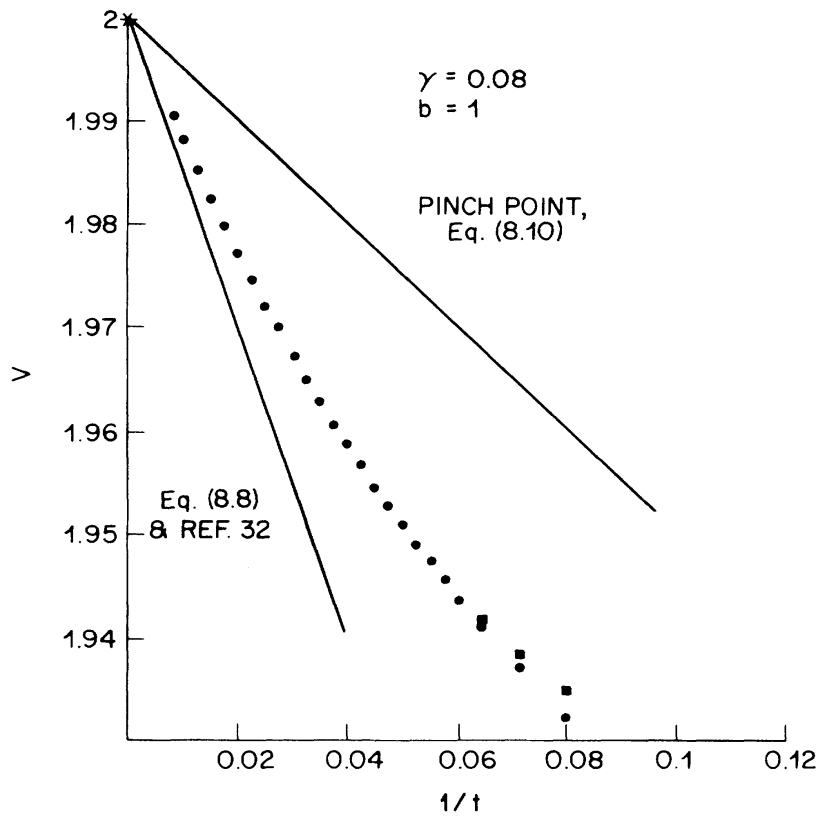

FIG. 17. Front velocity $v$ as a function of $1 / t$ for Eq. (4.1) with $b=1$. The dots are our data points measured at $\phi=0.05$, the squares the ones at $\phi=0.5$. The solid lines are the predictions of Eqs. (8.8) and (8.10). The simulations were performed with 3600 grid points with spacing 0.08 and time step 0.01 .

these simulations, I have also checked Bramson's result that the velocity converges uniformly in $x$, by comparing the front velocity observed at the point where $\phi(x, t)=0.05$ with the one at $\phi(x, t)=0.5$. The latter data are only plotted with squares for $t^{-1}>0.06$; for longer times, these data fall right on top of the dots. Data for Eq. (5.1) with $\gamma=0.08, b=1$ are presented in Fig. 18; although the convergence to the asymptotic result appears to be somewhat slower (possibly because for $\gamma$ close to $\frac{1}{12}$, three roots are nearly equal), the data are reasonably consistent with Eq. (8.8).

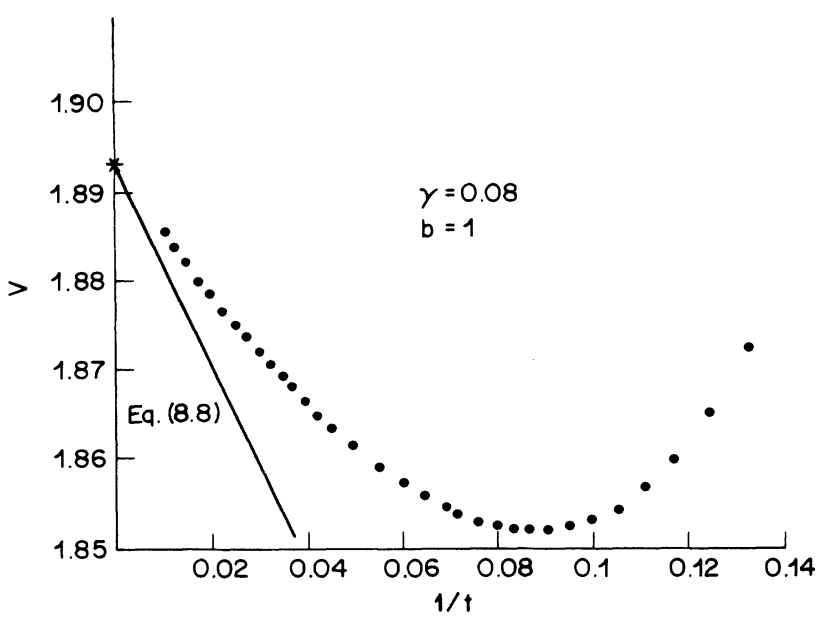

FIG. 18. As Fig. 17, but for Eq. (5.1) with $b=1$ and $\gamma=0.08$. The solid line is our asymptotic result (8.8).
We note finally that the convergence to the asymptotic velocity is very different in the nonlinear-marginalstability regime. Indeed, since the "invasion mode" responsible for nonlinear marginal stability corresponds to an isolated unstable mode for profiles with velocities less than $v^{\dagger}$, we generally expect an exponential relaxation towards $v^{\dagger}$,

$$
v=v^{\dagger}+D e^{-\Delta t},
$$

with a relaxation rate $\Delta$ which will depend on the initial conditions. For example, when the nonlinear-marginalstability profile overtakes a profile that falls off as $e^{i k_{1} x}$ and whose speed is $v_{1}$, a simple analysis in the tails shows that the relaxation rate is

$$
\Delta=k_{1}^{i}\left(v^{\dagger}-v_{1}\right) \text {. }
$$

To illustrate this, we plot in Fig. $19 \ln \left[v(t)-v^{\dagger}\right]$ versus $t$ from numerical solutions of Eq. (5.1) with $\gamma=0.08$, $b=0.25$, and with initial profiles that fall off as $e^{i k_{1} x}$. The behavior is clearly consistent with Eq. (8.11), and the measured values of the slope agree within about $5 \%$ with the values predicted from (8.12), except for the data at $k_{1}^{i}=0.78$ for which $v_{1}$ is close to $v^{\dagger}$, where the discrepancy is about $50 \%$. We think that this is due to the existence of long transients, as well as the discrepancy between the numerical value and predicted values of $v^{\dagger}$.

\section{SIMPLIFICATION OF THE OPERATOR $\mathcal{L}$ IN EQ. (2.3)}

In Ref. 9, I concentrated my analysis on the first term on the right-hand side of Eq. (2.2), the idea being that this would be correct as long as the linearized operator $\mathcal{L}$ would be stable. I pointed out that this stability could be checked explicitly for any given equation.

I have only realized later that, in fact, $\mathcal{L}$ has quite a simple structure: the requirement that $\mathcal{L}$ be stable is actually nothing but the requirement that we always pick

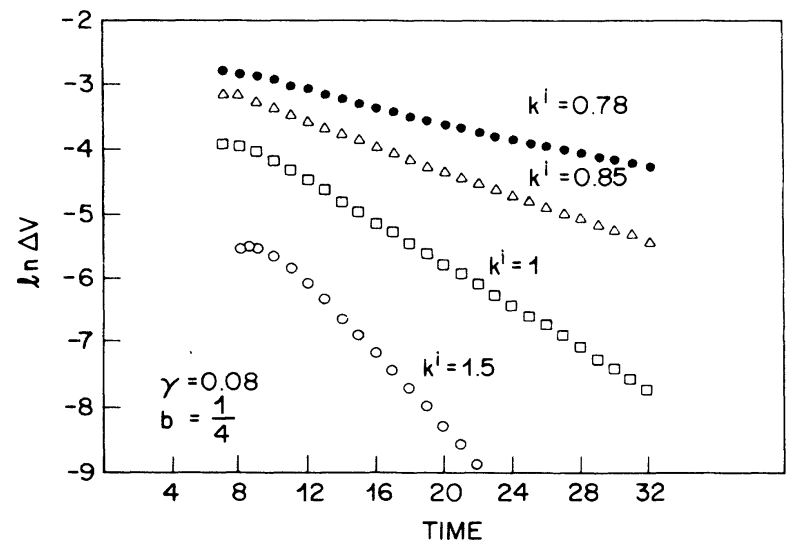

FIG. 19. Plot of $\ln \left[v(t)-v^{\dagger}\right]$ vs time to illustrate the exponential decay towards $v^{\dagger}$. The different data correspond to runs with different initial conditions, which were of the form $\phi \propto \exp \left(-k^{i} x\right)$, with $k^{i}$ as indicated. 
the absolute maximum of $\omega^{i}$ as a function of $k^{r}$ in solving (2.1b), $\partial \omega^{i} / \partial k^{r}=0$ for fixed $k^{r}$. With hindsight, it is obvious that some simplification should occur: ${ }^{50}$ essentially all necessary information about the linear dynamical equation we started out with, is contained in the dispersion relation $\omega(k)$. Although we perform a nonlinear transformation $\phi=e^{i u(x, t)}$ to bring out the general properties of the equation, the terms $\mathcal{L}$ should nevertheless be simply related to those of $\omega(k)$. We now show this explicitly.

When considering linear perturbations $\delta q$ around a solution $q=k$, we get for $\mathcal{L}$, upon transforming back to the (comoving) $x^{\prime}$ coordinates as in Sec. VIII,

$$
\mathcal{L} \delta q=-\left.f_{q_{x}}\right|_{q=k} \delta q_{x x}-f_{q_{x x x}} \delta q_{x x x}-\cdots .
$$

Since $\mathcal{L} q$ enters Eq. (2.2) in the form $q_{t}=\mathcal{L} q$, the requirement that the linearized operator be stable implies that for perturbation $\delta q \sim e^{i Q x}$ with $Q$ real, the eigenvalues $\Omega$ that satisfy

$$
-i \Omega=-\left.f_{q_{x}}\right|_{q=k} i^{2} Q^{2}-\left.f_{q_{x x}}\right|_{q=k} i^{3} Q^{3}-\cdots
$$

should have $\operatorname{Im} \Omega<0$ for all $Q$.

To arrive at the relations between the derivatives of $f$, consider the effect of the differential operator $\partial^{n} / \partial x^{n}$ in the dynamical equation. When we perform the transformation $\phi=e^{i u(x, t)}$, we generate terms involving $q=u_{x}$, $q_{x}$, etc. To evaluate (9.2), we need only the terms in $f$ linear in $q_{x}, q_{x x}$, etc. For the linear terms arising from the operator $\partial^{n} / \partial x^{n}$, we get

$$
\begin{aligned}
\frac{\partial^{n} e^{i u}}{\partial x^{n}}=e^{i u} & i^{n} q^{n}+\left(\begin{array}{l}
n \\
2
\end{array}\right) i^{n-1} q^{n-2} \frac{\partial q}{\partial x} \\
& +\left(\begin{array}{l}
n \\
3
\end{array}\right) i^{n-2} q^{n-3} \frac{\partial^{2} q}{\partial x^{2}}+\cdots \\
& \left.+\left(\begin{array}{l}
n \\
n
\end{array}\right) i \frac{\partial^{n-1} q}{\partial x^{n-1}}\right]+ \text { h.o. }
\end{aligned}
$$

Here,

$$
\left[\begin{array}{l}
n \\
k
\end{array}\right]=n ! /(n-k) ! k !
$$

and h.o. stands for terms of higher order in $q_{x}, q_{x x}$, etc. Equation (9.3) is easy to prove by induction with the help of the relation

$$
\left(\begin{array}{c}
n+1 \\
k
\end{array}\right)=\left(\begin{array}{l}
n \\
k
\end{array}\right)+\left(\begin{array}{c}
n \\
k-1
\end{array}\right) .
$$

By straightforward differentiation of (9.3), the following relations for the derivatives of $f$ immediately follow:

$$
\begin{aligned}
& f_{q_{x}}=\left.\frac{-i}{2 !} \frac{\partial^{2} f}{\partial q^{2}}\right|_{q=k}=\frac{-i}{2 !} \frac{d^{2} \omega}{d k^{2}} \\
& f_{q_{x x}}=\left.\frac{(-i)^{2}}{3 !} \frac{\partial^{3} f}{\partial q^{3}}\right|_{q=k}=\frac{(-i)^{2}}{3 !} \frac{d^{3} \omega}{d k^{3}}, \\
& \frac{\partial f}{\partial\left[\frac{\partial^{n} q}{\partial x^{n}}\right]}=\left.\frac{(-i)^{n}}{(n+1) !} \frac{\partial^{n} f}{\partial q^{n}}\right|_{q=k}=\frac{(-i)^{n}}{(n+1) !} \frac{d^{n+1} \omega}{d k^{n+1}}
\end{aligned}
$$

Using these relations in Eq. (9.2), we get

$$
\Omega=\sum_{n=2} \frac{1}{n !} \frac{d^{n} \omega}{d k^{n}} Q^{n},
$$

and since for an analytic dispersion relation $\omega(k)$ one has $\operatorname{Im}\left(d^{n} \omega / d k^{n}=\partial^{n} \omega^{i} /\left(\partial k^{r}\right)^{n}\right.$, we finally have

$$
\operatorname{Im} \Omega=\sum_{n=2} \frac{1}{n !} \frac{\partial^{n} \omega^{i}}{\partial\left(k^{r}\right)^{n}} Q^{n}
$$

Since the expression on the right-hand side is nothing but the deviation of the growth rate $\omega^{i}$ from the extremum at which $\partial \omega^{i} / \partial k^{r}=0$ for arbitrary changes $Q$ in $k^{r}$, the requirement that $\mathcal{L}$ be stable for all $Q$, amounts to the statement that the relevant solution is the wave number $k^{r}$ corresponding to the absolute maximum of $\omega^{i}$ (for fixed $k^{r}$ ). This choice already underlies our picture, and hence the condition on $\mathcal{L}$ is essentially trivial. ${ }^{51}$ of course, we could go even further and also relate the nonlinear terms in $\mathcal{L}$ to the properties of $\omega(k)$ by further undoing the nonlinear transformation to the variable $u(x, t)$, but we see no particular reason to do so.

\section{CONCLUDING REMARKS}

In this paper, I have shown that the transition from linear marginal stability to nonlinear marginal stability can be readily described within our dynamical picture for front propagation into unstable states. The resulting approach yields a physical interpretation and extension of the results of Aronson and Weinberger ${ }^{1}$ and Ben-Jacob et al. ${ }^{3}$ A transition to nonlinear marginal stability occurs when the linear-marginal-stability profile becomes unstable to a nonlinear invasion mode. For uniformly translating front profiles of the form $\phi(x-v t)$, the connection between stability and velocity selection can be demonstrated quite convincingly in general. While our physical picture and numerical calculations support the idea that the same dynamical mechanism controls velocity selection in general pattern-forming systems, I do not know how to prove this mathematically. As a first step towards this goal, it would be useful to extend the analysis of Collet and Eckmann ${ }^{6}$ of the Swift-Hohenberg equation [Eq. (5.3) with $b=0$ ] to the modified versions of this equation that exhibit nonlinear marginal stability.

As we have illustrated with two examples, close to the threshold for instability of pattern-forming systems, detailed predictions can be made by inspection of the proper amplitude equation. When the bifurcation describing the onset of instability is a pitchfork bifurcation, front propagation into the unstable state close to threshold is 
determined by whether the bifurcation is subcritical (inverted) or supercritical (forward). If the transition is of first order (corresponding to a subcritical bifurcation), front propagation into unstable states is generally governed by nonlinear marginal stability, while if the transition is continuous (corresponding to a supercritical bifurcation), linear marginal stability holds.

Have all possible mechanisms of front propagation into unstable states now been identified? In other words, is it the case that the propagation of fronts emerging from sufficiently localized initial conditions is governed by either the linear or the nonlinear-marginal-stability mechanism? From the work of Aronson and Weinberger, ${ }^{1}$ we know that the answer to this question is affirmative for second-order partial differential equations of the type (3.3); within our picture, it is hard to see how one could get a velocity selection other than through linear or nonlinear marginal stability, suggesting that these are indeed the two generic mechanisms. Again, however, it remains to be proven whether or not this is true.

To my knowledge, the only published experiments in which the predictions of Aronson and Weinberger ${ }^{1}$ for Eq. (3.3) have been tested experimentally in the regime we refer to as nonlinear marginal stability, are those of Hanna et al. ${ }^{17}$ on a chemical wave in an iodate-arsenous acid system. The composition wave in their experiments is accurately modeled by an equation of the type (4.1) in the limit $b<<1$. In this limit one is far into the nonlinear-marginal-stability regime, so that $v^{\dagger}$ is a lot larger than $v^{*}$. The observed wave speeds are consistent with the value of $v^{\dagger}$ for their parameters, and hence their experiment can be viewed as providing a direct and accurate test of what we refer to as nonlinear marginal stability.

I believe that the most important implications of this work will turn out to be for various pattern-forming instabilities found in fluid dynamics and other fields. Experiments on pattern-forming systems that focus specifically on front propagation into unstable states are scarce: the only two I am aware of are those of Fineberg and Steinberg, ${ }^{13}$ who found their fronts in a RayleighBénard cell to agree with the linear-marginal-stability predictions, and those of Ahlers and Cannell ${ }^{11}$ on vortex fronts in Taylor-Couette flow. The front speeds they measured were smaller than $v^{*}$, and therefore were neither consistent with linear nor with nonlinear marginal stability (since $v^{\dagger}>v^{*}$ ). Neither can their results be attributed to initial conditions with too slow spatial decay, since this should also give rise to velocities larger than $v^{*}$. Extensive numerical studies ${ }^{12}$ of the full hydrodynamic equations always give results consistent with linear marginal stability, and thus have failed to resolve this discrepancy.

The difficulty in performing the above experiments lies in preparing the system in the unstable state: since both the Rayleigh-Bénard and the Taylor-Couette instability correspond to a stationary bifurcation, this had to be done by suddenly changing the control parameter. At a convective instability, on the other hand, a system is unstable, but the growing perturbation is convected away fast enough that the disturbance is not growing locally.
As a result, unstable systems can occur naturally - i.e., without the need of careful experimental control-near convective instabilities. The following examples illustrate the relevance of our work for such systems.

(i) Rayleigh-Bénard convection in binary fluid mixtures is at present under active experimental and theoretical investigation, ${ }^{26,27,52}$ because of the rich variety of standingas well as traveling-wave patterns observed. It is well known ${ }^{52}$ that the transition to the traveling-wave pattern is subcritical for almost all parameter values. It would therefore be extremely interesting to extend the earlier front propagation experiments of Fineberg and Steinberg ${ }^{13}$ to binary mixtures, as our arguments imply that the fronts near the transition should be governed by nonlinear marginal stability. They should thus propagate as a finite speed $v^{\dagger}$ much larger than the velocity $v^{*}$ which becomes vanishingly small at the transition.

Since traveling-wave patterns have a nonzero group velocity, Cross ${ }^{26}$ has argued that in a finite cell the nature of the convective state is intimately connected with the front-propagation behavior. Cross predicts and verifies numerically for a model amplitude equation with saturating nonlinearities (corresponding to a supercritical bifurcation), that when the group velocity $s$ of the waves is less than the linear-marginal-stability value $v^{*}$, an extended traveling-wave pattern is established in the whole cell (in this case the conductive state is absolutely unstable). For $s>v^{*}$, on the other hand, only confined regions of traveling waves exist in his model, since the conductive state is then only convectively unstable. Since in reality the experiments exhibit a subcritical bifurcation, ${ }^{27,52}$ one would expect the relevant criterion for the pattern selection to be whether $s$ is larger or smaller than $v^{\dagger}$ (as always, our picture implies $v^{\dagger}>v^{*}$ ). However, recent experiments by Fineberg et al. ${ }^{27}$ appear to give a crossover at a value of $s$ which is somewhat less than $v^{*}$. Clearly, more work is needed to understand this discrepancy. ${ }^{53}$

(ii) As is long since known, flow of a fluid past a cylinder leads to the creation of a von Kármán vortex street at sufficiently high Reynolds numbers. ${ }^{54}$ It was, however, only recently that the transition to vortex creation was argued ${ }^{23}$ to be associated with a linear absolute instability in the wake behind the cylinder. This prediction was based on a linear-stability analysis of the equations in the wake with the aid of the "pinch-point" analysis of Bers. ${ }^{22}$ As we have discussed in the Introduction, this is equivalent to what $I$ refer to as a linearmarginal-stability analysis. The work in this paper thus shows that the argument of Triantafyllou et al. ${ }^{23}$ is only self-consistent if the wake instability is supercritical. Fortunately, velocity fluctuations in the wake studied with laser Doppler anemometry by Mathis et al. ${ }^{55}$ are in agreement with the bifurcation being supercritical. Hence the experiments nicely justify the assumption underlying the theoretical analysis, and together, theory and experiment provide an elegant and consistent picture of the onset of the von Kármán vortex street. Ideas similar to those described above have been applied to the general problem of vortex shedding of bluff bodies. ${ }^{25}$

(iii) Experimentally, plane Poiseuille flow is often found to become unstable ${ }^{56}$ at Reynolds numbers $R \gtrsim 1000$, 
whereas early stability analysis ${ }^{57}$ predicted the basic parabolic profile to be linearly stable up to $R$ of about 5770 . Moreover, recent investigations ${ }^{58}$ show that the flow is linearly only convectively unstable for all $R \geq R_{c}$ $=5772.22$. Clearly, the experimental instability must be due to nonlinearities, a suggestion first made ${ }^{59}$ almost 40 years ago; indeed, Stewartson and Stuart ${ }^{60}$ later showed that the instability at $R_{c}$ corresponds to a subcritical bifurcation, and subsequent analytical ${ }^{61}$ and numerical ${ }^{62}$ work has confirmed the nonlinear nature of the instability of plane Poiseuille flow. Although it is still under investigation whether the approach in this paper can provide new insight into this problem, it should be clear that this example, when rephrased in the context of front propagation, conforms to the unified picture advocated here: since the bifurcation is subcritical, nonlinear fronts propagating into the unstable parabolic velocity profile for $R$ near $R_{c}$ will propagate with speed $v^{\dagger}$, not $v^{*}$, as assumed in a linear analysis. If it turns out that $v^{\dagger}$ is large, the flow is absolutely unstable against the intrusion by nonlinear fronts, even though it is only convectively unstable ${ }^{58}$ linearly.

The above examples not only illustrate that nonlinear marginal stability may be of immediate importance to experiments on binary mixtures, but also that the viewpoint advocated here puts several other instabilities in fluid dynamics in a new perspective. We hope that this work will stimulate further studies of these questions.

\section{ACKNOWLEDGMENTS}

I would like to acknowledge stimulating collaborations with Pat Cladis and Greg Dee that inspired the work described here. Also, I am grateful to Greg Dee for his help in getting started with the numerics, and especially to Pierre Hohenberg for numerous discussions and comments on the subject.

\section{APPENDIX A: INSTABILITY OF FRONTS WITH $v<v^{\dagger}$}

In this appendix we demonstrate that the point where $v=v^{\dagger}$ is also a marginal stability point for uniformly translating profiles $\phi(x-v t)$. For equations of the type (3.3), the stability analysis can be done explicitly by transforming the linearized equation to a Schrödinger-type equation; ${ }^{3}$ the argument given here holds for any translation-invariant equation that admits uniformly translating profiles.

Consider a dynamical equation of the form (in case of more than one field, $\phi$ denotes a vector)

$$
\frac{\partial \phi}{\partial t}+g_{1} \frac{\partial^{2} \phi}{\partial t^{2}}+\cdots=F\left(\phi, \phi_{x}, \phi_{x x}, \ldots\right) .
$$

The uniformly translating profile $\phi_{u}(x-v t)$ then obeys the ordinary differential equation

$$
-v \frac{d \phi_{u}}{d x}+g_{1} v^{2} \frac{d^{2} \phi_{u}}{d x^{2}}+\cdots=F\left[\phi_{u}, \frac{d \phi_{u}}{d x}, \frac{d^{2} \phi_{u}}{d x^{2}}, \ldots\right] \text {, }
$$

and in the frame $x^{\prime}=x-v t$ moving with velocity $v$, linear perturbation in $\phi$ of the form $\Psi\left(x^{\prime}\right) e^{\Omega t}$ obeys

$$
\left(\Omega+g_{1} \Omega^{2}+\cdots\right) \Psi-v \frac{d \Psi}{d x^{\prime}}+g_{1} \frac{d^{2} \Psi}{d x^{2}}+\cdots=G \Psi,
$$

where $G$ is a linear operator obtained by linearizing $\phi$ in $F$ around $\phi_{u}$. For large $x, \phi_{u}$ and $\Psi$ will be of the form

$$
\begin{gathered}
\phi_{u}=\sum_{j} C_{j} e^{i k_{j} x^{\prime}}, \quad 0<\operatorname{Im} k_{j}<\operatorname{Im} k_{j+1}, \\
\Psi=\sum_{j} B_{j} e^{i K_{j} x^{\prime}}, \quad 0<\operatorname{Im} K_{j}<\operatorname{Im} K_{j+1},
\end{gathered}
$$

where the sums run over all roots $k_{j}$ and $K_{j}$ with a positive imaginary part. For physical reasons, we only allow sufficiently localized perturbation of $\phi$. Thus $\Psi$ has to fall off faster than $\phi_{u}$ for large $x$. Note that $K_{1}$ is the root $K$, with the smallest imaginary part corresponding to the slowest spatial falloff. In view of the counting argument of Appendix A of Ref. 9 and the fact that (A3) is linear in $\Psi$, there will in general be one particular eigenvalue $\Omega^{\prime}(v)$ that satisfies the additional constraint $B_{1}=0$; since $B_{1}=0$, the corresponding eigenfunction will obey the boundary condition that $\Psi$ decay faster than $\phi_{u}$, at least when $\left|\Omega^{\prime}\right|$ is small enough so that $\operatorname{Im} K_{2}>\operatorname{Im} k_{1}$.

Because of translation invariance of Eq. (A1), Eq. (A3) will always admit a zero eigenmode. For this mode, then, we have $K_{j}=k_{j}$ and $B_{j}=C_{j}$. Thus, since the velocity $v^{\dagger}$ just marks the point where the coefficient $C_{1}$ vanishes [cf. Eq. (3.2)], the eigenfunction defined by $B_{1}=0$ has to coincide with the translation mode, and $\Omega^{\prime}\left(v^{\dagger}\right)=0$. Thus, since $\Omega^{\prime}$ goes through zero at $v=v^{\dagger}$, either the front solutions for $v>v^{\dagger}$ or those for $v<v^{\dagger}$ will be unstable. The discussion in Sec. III shows that the instability occurs for $v<v^{\dagger}$.

\section{APPENDIX B: SUMMARY OF STABILITY - ANALYSIS ARGUMENTS}

In this appendix, I expound on the remarks made in Sec. III concerning the stability of the nonlinearmarginal-stability profile $\phi\left(x-v^{\dagger} t\right)$. As in Appendix A, I will restrict myself to the case of uniformly translating profiles. For the special case of Eq. (3.3), see also the paper by Ben-Jacob et al. ${ }^{3}$

First consider the stability of arbitrary profiles $\phi(x-v t)$ with $v \neq v^{\dagger}$. According to the counting argument of Ref. 9, Appendix A, almost all eigenfunctions $\Psi$ obeying Eq. (A3) will have $B_{1} \neq 0$ [cf. Eq. (A4)]. With the condition $K_{1}^{i}>k_{1}^{i}$ in Eq. (A4) the behavior of these eigenfunctions with $B_{1}=0$ can be obtained by comparing the dynamics in the leading edge only. The stability of these modes follows from the fact that $v_{\mathrm{gr}}<v$ on the branch drawn with a solid line in Fig. 2 (cf. also Ref. 9, Sec. III B), and is illustrated graphically in Fig. 3. The particular eigenfunction with $B_{1}=0$ and $\Omega=\Omega^{\prime}$ has been analyzed in Appendix A. We have shown that $\Omega^{\prime}$ goes through zero at $v^{\dagger}$ and argued that this implies that profiles with $v<v^{\dagger}$ are unstable to this invasion mode (cf. Fig. 4). The reason that the argument that $v_{\mathrm{gr}}<v$ implies 
stability does not apply here is already apparent from Fig. 4(c): the asymptotic behavior of the mode with $B_{1}=0$ corresponds to a point on the different branch of the curve $v\left(k^{i}\right)$, and cannot be obtained by linearizing the local wave number $q$ around $k_{1}$.

The stability of the nonlinear-marginal-stability profile $\phi\left(x-v^{\dagger} t\right)$ follows from an essentially similar consideration. Again, almost all eigenfunctions $\Psi$ have $B_{1} \neq 0$. With the requirement that these satisfy $K_{1}^{i}>k_{1}^{i}\left(v=v^{\dagger}\right)$ it is easy to convince oneself with a construction as in Fig. 4(c) that $\phi\left(x-v^{\dagger} t\right)$ is stable with respect to such perturbations. Note again that linearizing $q$ about $k_{2}^{\dagger}$ and concluding that the front profile is unstable, in view of the fact that $v_{\mathrm{gr}}>v$ along the dashed branch in Fig. 4(a), is incorrect, since the perturbation with $B_{1} \neq 0$ corresponds to large perturbations in $q^{i}$ on a different branch of $v\left(k^{i}\right)$. Of course, there is also a mode with $B_{1}\left(v=v^{\dagger}\right)=0$. However, this must be the zero eigenmode corresponding to translation invariance, and hence the nonlinear-marginal-stability profile $\phi\left(x-v^{\dagger} t\right)$ is stable.

\section{APPENDIX C: DERIVATION OF EQS. (8.6) AND (8.8) FROM THE PINCH-POINT ANALYSIS}

In this appendix I show that terms that are commonly neglected in the pinch-point analysis ${ }^{22,29}$ appear to lead to the proper asymptotic expressions (8.6) and (8.8) for the local wave number and velocity. The analysis therefore indicates that the usual expression (8.9) is not valid for all $x$, an observation already made by Lifshitz and Pitaevskii. ${ }^{29}$

Apart from some slight changes in notation, we will essentially follow Bers. ${ }^{22}$ In the comoving frame $x^{\prime}=x-v^{*} t$, we want to derive the asymptotic expression for the Green's function $G\left(x^{\prime}, t\right)$, which is given by

$$
G\left(x^{\prime}, t\right)=\frac{1}{2 \pi} \int_{\tilde{L}} d \omega e^{i \omega t} I\left(x^{\prime}, \omega\right),
$$

with [cf. Bers, ${ }^{22}$ Eq. (19)]

$I\left(x^{\prime}, \omega\right)=i \Theta\left(x^{\prime}\right) \sum_{k_{u}} \frac{e^{-i k_{u}(\omega) x^{\prime}}}{\partial D /\left.\partial k\right|_{k_{u}}}-i \Theta\left(-x^{\prime}\right) \sum_{k_{l}} \frac{e^{i k_{l}(\omega) x^{\prime}}}{\partial D /\left.\partial k\right|_{k_{l}}}$.

Here $\widetilde{L}$ is some contour in the $\omega$ plane, $\Theta(x)$ is the unit step function, and $D=D(\omega, k)$ is a function of $\omega$ and $k$ whose zeros determine the dispersion relation $\omega(k)$ in the comoving frame

$$
D(\omega, k)=0 \rightarrow \omega=\omega(k) .
$$

The first (second) sum in (C2) is over all the poles $k_{u}\left(k_{l}\right)$ that are in the upper (lower) half plane for $\omega \rightarrow i \infty$. It is argued $^{22,29}$ that the asymptotic behavior of $G$ in $(\mathrm{C} 1)$ is determined by the point in the complex plane where a root $k_{u}$ and a root $k_{l}$ coalesce. This happens at a point $\omega^{*}, k^{*}$ where $D(\omega, k)=0$ has two double roots, or $D\left(\omega^{*}, k^{*}\right)=0, \partial D /\left.\partial k\right|_{k^{*}}=0$. Using Eq. (C3), it is easy to see that this coincides with our marginal-stability equation (2.1) in the comoving frame. Near this point, we may write [cf. Bers, ${ }^{22}$ Eqs. (22)-(24)]

$\left(k-k^{*}\right)^{2}=C^{2}\left(\omega-\omega^{*}\right)-2 E\left(\omega-\omega^{*}\right)\left(k-k^{*}\right)+\cdots$,

$D(\omega, k)=\left(k-k_{u}(\omega)\right)\left(k-k_{l}(\omega)\right) R(\omega, k)$,

so that

$$
\begin{aligned}
& k_{u}=k^{*}+C\left(\omega-\omega^{*}\right)^{1 / 2}-E\left(\omega-\omega^{*}\right)+\cdots, \\
& k_{l}=k^{*}-C\left(\omega-\omega^{*}\right)^{1 / 2}-E\left(\omega-\omega^{*}\right)+\cdots .
\end{aligned}
$$

Note that in (C4) we can make the identifications

$$
C^{2}=2 /\left.\frac{d^{2} \omega}{d k^{2}}\right|_{*}, \quad E=\left.\frac{1}{3} C^{4} \frac{d^{3} \omega}{d k^{3}}\right|_{*} .
$$

With these results, we can write for $(\mathrm{C} 2)$

$$
\begin{aligned}
I\left(x^{\prime}, \omega\right)= & \frac{i \Theta(x) e^{i\left[k^{*}+C\left(\omega-\omega^{*}\right)^{1 / 2}-E\left(\omega-\omega^{*}\right)\right] x^{\prime}}}{2 C\left(\omega-\omega^{*}\right)^{1 / 2} R^{*}\left[1+S\left(\omega-\omega^{*}\right)^{1 / 2}+\cdots\right]} \\
& +\frac{i \Theta(-x) e^{i\left[k^{*}-C\left(\omega-\omega^{*}\right)^{1 / 2}-E\left(\omega-\omega^{*}\right)\right] x^{\prime}}}{2 C\left(\omega-\omega^{*}\right)^{1 / 2} R^{*}\left[1-S\left(\omega-\omega^{*}\right)^{1 / 2}+\cdots\right]},
\end{aligned}
$$

with

$$
\left.S \equiv \frac{C}{R^{*}} \frac{\partial R}{\partial k}\right|_{k^{*}}
$$

To evaluate $G$, let us for concreteness take $x^{\prime}>0$ (the case $x^{\prime}<0$ can be worked out analogously). Upon substitution of (C8) for $x^{\prime}>0$ into (C1), we get upon expanding the term in the denominator

$$
G\left(x^{\prime}, t\right) \simeq \frac{i e^{i k^{*} x^{\prime}-i \omega^{*} t}}{4 \pi C R^{*}} \int_{L} d \omega \frac{1-S\left(\omega-\omega^{*}\right)^{1 / 2}}{\left(\omega-\omega^{*}\right)^{1 / 2}} e^{i C\left(\omega-\omega^{*}\right)^{1 / 2} x^{\prime}-i(E x+t)\left(\omega-\omega^{*}\right)}
$$

where the $\widetilde{L}$ is around the branch cut at $\omega^{*}$. Upon neglecting the terms proportional to $S, C$, and $E$, this expression reduces to the one usually given, ${ }^{22,29}$ which in turn leads to the result (8.9). The present expression is the one needed to arrive at the proper results (8.6) and (8.8).
Let us take the branch cut along the line where $\arg \left(\omega-\omega^{*}\right)=3 \pi / 2$, and for simplicity assume that $E x^{\prime}+t$ is real and positive (the extension to the general case is straightforward). By transforming to the variable $u=e^{i \pi / 4}\left(E x^{\prime}+t\right)^{1 / 2}\left(\omega-\omega^{*}\right)^{1 / 2}$, we then obtain from (C10) 


$$
\begin{aligned}
G\left(x^{\prime}, t\right) \approx & \frac{e^{i k^{*} x^{\prime}-i \omega^{*} t+i \pi 4}}{2 \pi C R^{*}\left(E x^{\prime}+t\right)^{1 / 2}} e^{i C^{2} x^{\prime 2} / 4\left(E x^{\prime}+t\right)} \\
& \times\left[1+\frac{S C x^{\prime}}{2\left(E x^{\prime}+t\right)}\right] .
\end{aligned}
$$

In the limit $t \rightarrow \infty, x^{\prime}$ fixed, this result agrees with the one usually obtained, Eq. (8.9). However, if we assume that the second term in large parentheses is the dominant one and put this term in the exponent, we get for the deviation $\rho$ of the local wave vector from the value $k^{*}$

$$
\begin{aligned}
\rho\left(x^{\prime}, t\right) & \simeq-\frac{i}{x^{\prime}}+\frac{1}{2} \frac{C^{2} x^{\prime}}{t} \quad\left[\frac{x^{\prime}}{t} \ll 1\right) \\
& =-\frac{i}{x^{\prime}}+\left(\left.\left.\frac{d^{2} \omega}{d k^{2}}\right|_{*}\right|^{-1} \frac{x^{\prime}}{t},\right.
\end{aligned}
$$

where we used Eq. (C7). This result is equivalent to Eq. (8.6). Similarly, because the $t^{-3 / 2}$ prefactor of the second term in large parentheses in Eq. (C11) leads to a $-\frac{3}{2} \ln t$ term in the exponent, one recovers the result (8.8).

The above analysis illustrates that we also arrive at our asymptotic expressions (8.6) and (8.8) if we view the second contribution to the term between parentheses in (C11) as the dominant one. Nevertheless, the analysis is still not totally satisfactory, as this term is formally only of order unity if $x^{\prime} / t=O(1)$, and is only large for $x \rightarrow \infty$ if $E$ is sufficiently small ( $E=0$ for the two-model equation that we studied numerically). I have not been able to resolve this issue any further.

\section{APPENDIX D: AMPLITUDE EQUATION EXPANSION}

In the appendix we follow Cross et $a l .{ }^{45}$ in deriving an amplitude equation expansion for the generalized SwiftHohenberg equation,

$$
\frac{\partial u}{\partial t}=\left[\epsilon-\left(\partial_{x}^{2}+q_{0}^{2}\right)^{2}\right] u+b u^{2}+d u^{3}-e u^{5} .
$$

For $q_{0}=1, d=-1, e=0$, this equation is equivalent to Eq. (5.4), and for $q_{0}=1, b=0, e=1$, to Eq. (5.9). Throughout this appendix, I will choose my notation to follow as closely as possible the one used by Cross et $a l .,{ }^{45}$ who studied the case $b=e=0, d=-1$.

Our goal is to derive the first few terms in an expansion for $u$ of the form

$$
u=\epsilon^{1 / 2} u_{0}+\epsilon u_{1}+\epsilon^{3 / 2} u_{2}+\epsilon^{2} u_{3}+\cdots
$$

with the functions $u_{i}$ separated into rapidly varying parts $\exp \left(i n q_{0} x\right)$ and slowly varying parts, depending on the slow variables $X=\epsilon^{1 / 2} x, T=\epsilon t$,

$$
\begin{aligned}
u_{i}= & O_{i}(X, T)+\frac{1}{\sqrt{3}}\left[A_{i}(X, T) e^{i q_{0} x}+\text { c.c. }\right] \\
& +\frac{1}{\sqrt{3}}\left[B_{i}(X, T) e^{2 i q_{0} x}+\text { c.c. }\right] \\
& +\frac{1}{\sqrt{3}}\left[C_{i}(X, T) e^{3 i q_{0} x}+\text { c.c. }\right]+\cdots
\end{aligned}
$$

(Cross et al. ${ }^{45}$ use $B_{i}$ for my $C_{i}$.) From this expansion, we then identify the coefficients in Eq. (6.4) for the complex amplitude function

$\Phi(X, T) \equiv \epsilon^{1 / 2} A_{0}(X, T)+\epsilon A_{1}(X, T)+\epsilon^{3 / 2} A_{2}(X, T)+\cdots$.

(D4)

To arrive at the expansion, one divides the gradient $\partial_{x}$ into two parts, ${ }^{45}$

$$
\partial_{x} \rightarrow \partial_{x}+\epsilon^{1 / 2} \partial_{X},
$$

with the first term acting only on the rapidly varying part. The differential operator in (D1) then becomes

$$
\begin{aligned}
\left(\partial_{x}^{2}+q_{0}^{2}\right)^{2} \rightarrow & \square^{2}+4 \epsilon^{1 / 2} \partial_{x} \partial_{X} \square+\epsilon\left(6 \partial_{x}^{2} \partial_{X}^{2}+2 q_{0}^{2} \partial_{X}^{2}\right) \\
& +4 \epsilon^{3 / 2} \partial_{x} \partial_{X}^{3}+\epsilon^{2} \partial_{X}^{4},
\end{aligned}
$$

where $\square=\partial_{x}^{2}+q_{0}^{2}$ acts only on the rapidly varying part. Upon substitution of these expansions into Eq. (D1), we get

$$
\begin{aligned}
& \square^{2} u_{0}=0 \text { for } O\left(\epsilon^{1 / 2}\right) \text {, } \\
& \square^{2} u_{1}+4 \partial_{x} \partial_{X} \square u_{0}-b u_{0}^{2}=0 \text { for } O(\epsilon) \text {, } \\
& \partial_{T} u_{0}+\square^{2} u_{2}+4 \partial_{x} \partial_{X} \square u_{1}+\left(6 \partial_{x}^{2} \partial_{X}^{2}+2 q_{0}^{2} \partial_{X}^{2}\right) u_{0} \\
& -\left(d u_{0}^{2}+1\right) u_{0}-2 b u_{0} u_{1}=0 \text { for } O\left(\epsilon^{3 / 2}\right) \text {, } \\
& \partial_{T} u_{1}+\square^{2} u_{3}+4 \partial_{x} \partial_{X} \square u_{2}+\left(6 \partial_{x}^{2} \partial_{X}^{2}+2 q_{0}^{2} \partial_{X}^{2}\right) u_{1} \\
& +4 \partial_{x} \partial_{X}^{3} u_{0}-2 b u_{0} u_{2}-b u_{1}^{2}-\left(3 d u_{0}^{2}+1\right) u_{1} \\
& \text { for } O\left(\epsilon^{2}\right) \text {, } \\
& \partial_{T} u_{2}+\square^{2} u_{4}+4 \partial_{x} \partial_{X} \square u_{3}+\left(6 \partial_{x}^{2} \partial_{X}^{2}+2 q_{0}^{2} \partial_{X}^{2}\right) u_{2} \\
& +4 \partial_{x} \partial_{X}^{3} u_{1}+\partial_{X}^{4} u_{0}-2 b u_{0} u_{3}-2 b u_{1} u_{2}-\left(3 d u_{0}^{2}+1\right) u_{2} \\
& -3 d u_{1}^{2} u_{0}+e u_{0}^{5}=0 \text { for } O\left(\epsilon^{5 / 2}\right) \text {. }
\end{aligned}
$$

Since $\square \exp \left(i q_{0} x\right)=0$, Eq. (D7) shows that for $u_{0}$, only $A_{0}(X, T)$ can be nonzero; all other terms in (D3), $O_{0}, B_{0}$, etc., have to vanish. Then, (D8) is solved for arbitrary $A_{1}$ and by taking

$$
B_{1}=\frac{\sqrt{3} b}{27 q_{0}^{4}} A_{0}^{2}, \quad O_{1}=\frac{2 b \sqrt{3}}{3 q_{0}^{4}}\left|A_{0}\right|^{2},
$$

with higher-order terms $\left(C_{1}, D_{1}\right.$, etc. $)$ equal to zero. Since the term involving $A_{2}$ does not give any contribution in (D9), the requirement that the terms involving $\exp \left(i q_{0} x\right)$ satisfy (D9) gives the first nontrivial solvability condition,

$$
\begin{aligned}
\partial_{T} A_{0}-4 q_{0}^{2} \partial_{X}^{2} A_{0}-A_{0}-d & \left|A_{0}\right|^{2} A_{0} \\
& -\frac{2 b}{\sqrt{3}}\left(O_{1} A_{0}+A_{0}^{*} B_{1}\right)=0 .
\end{aligned}
$$

Upon introducing $\bar{X}=\left(2 q_{0}\right)^{-1} X$ and using (D12), this becomes

$$
\frac{\partial A_{0}}{\partial T}=\frac{\partial^{2} A_{0}}{\partial \bar{X}^{2}}+A_{0}+\left(d+\frac{38}{27} \frac{b^{2}}{q_{0}^{4}}\right)\left|A_{0}\right|^{2} A_{0} .
$$

The only other nonzero terms in the expansion of $u_{2}$ are, according to (D9), 
$O_{2}=\frac{2 b}{q_{0}^{4} \sqrt{3}}\left(A_{0} A_{1}^{*}+A_{0}^{*} A_{1}\right)$,

(D14)

$B_{2}=\frac{1}{9 q_{0}^{4}}\left(12 i q_{0}^{2} \partial_{\bar{X}} B_{1}+\frac{2 b}{\sqrt{3}} A_{0} A_{1}\right)$,

$$
=\frac{8 b \sqrt{3}}{81 q_{0}^{6}} i A_{0} \partial_{\bar{X}} A_{0}+\frac{2 b \sqrt{3}}{27 q_{0}^{4}} A_{0} A_{1} \text {, }
$$

$C_{2}=\frac{1}{192 q_{0}^{4}}\left(d A_{0}^{3}+2 b \sqrt{3} A_{0} B_{1}\right)=\frac{1}{192 q_{0}^{4}}\left(d+\frac{2 b^{2}}{9 q_{0}^{4}}\right) A_{0}^{3}$.

(D16)

I now turn to (D10). The solvability condition for $A_{1}$ reads

$$
\begin{array}{r}
\partial_{T} A_{1}-4 q_{0}^{4} \partial_{x}^{2} A_{1}+4 i q_{0} \partial_{x}^{3} A_{0}-\frac{2 b}{\sqrt{3}} A_{0} O_{2}-\frac{2 b}{\sqrt{3}} A_{0}^{*} B_{2} \\
-\frac{2 b}{\sqrt{3}}\left(A_{1} O_{1}+B_{1} A_{1}^{*}\right)-2 d\left|A_{0}\right|^{2} A_{1}-A_{1}=0 .
\end{array}
$$

With the aid of Eqs. (D12), (D14), and (D15), this becomes

$$
\begin{aligned}
\frac{\partial A_{1}}{\partial T}= & \frac{\partial^{2} A_{1}}{\partial \bar{X}^{2}}-\frac{i}{2 q_{0}^{2}} \frac{\partial^{3} A_{0}}{\partial \bar{X}^{3}}+\frac{16 i b^{2}}{81 q_{0}^{6}}\left|A_{0}\right|^{2} \frac{\partial A_{0}}{\partial \bar{X}}+A_{1} \\
& +\left(d+\frac{38 b^{2}}{27 q_{0}^{4}}\right)\left(A_{0}^{2} A_{1}^{*}+2\left|A_{0}\right|^{2} A_{1}\right)
\end{aligned}
$$

(D17)
If we write $\Phi$ in (D4) in terms of $\bar{X}$ and $T$ and substitute this expansion into Eq. (6.4), Eqs. (D13) and (D17) show that one should make the identifications

$$
\begin{aligned}
& c_{1}=d+\frac{38 b^{2}}{27 q_{0}^{2}}, \quad k_{2}=\frac{1}{2 q_{0}^{2}}, \\
& k_{4}=\frac{16 b^{2}}{81 q_{0}^{6}}, \quad k_{1}=k_{3}=k_{5}=0 .
\end{aligned}
$$

We see that $k_{4} \neq 0$ for $b \neq 0$. As explained in the text, this implies that the amplitude equation does not become of the form (6.1) in the transition region where $c_{1}=O\left(\epsilon^{1 / 2}\right)$. As a result, I cannot predict the transition value exactly to order $\epsilon^{1 / 2}$ for $b \neq 0$.

With $b \neq 0$, the number of terms rapidly proliferates: it is easy to see that $O_{3}, B_{3}, C_{3}$, and $D_{3}$ are all nonzero and given by rather complicated expressions. However, the only coefficient left to calculate is $c_{2}$ in Eq. (6.4), and to do so, I only need to get the prefactor of the terms $\left|A_{0}\right|^{4} A_{0}$ in the solvability condition for $A_{3}$ that one obtains from (D11). Only powers of $A_{0}$ and $A_{0}^{*}$ in $O_{3}, B_{3}$, $C_{3}$, and $D_{3}$ can contribute to this term; retaining only these contributions, I then find from (D10)

$$
\begin{aligned}
O_{3} & =\frac{1}{q_{0}^{4}}\left(\frac{b}{\sqrt{3}} O_{1}^{2}+\frac{2 b}{\sqrt{3}}\left|B_{1}\right|^{2}+d\left(A_{0}^{2} B_{1}^{*}+A_{0}^{* 2} B_{1}+2\left|A_{0}\right|^{2} O_{1}\right)+O_{1}-\partial_{T} O_{1}+\cdots\right) \\
& =\left(\frac{2 \sqrt{3} b d}{27 q_{0}^{8}}-\frac{1042 b^{3} \sqrt{3}}{729 q_{0}^{12}}\right)\left|A_{0}\right|^{4}-\frac{2 b \sqrt{3}}{3 q_{0}^{8}}\left|A_{0}\right|^{2}+\text { o.t. }, \\
B_{3} & =\frac{1}{9 q_{0}^{4}}\left(-\partial_{T} B_{1}+\frac{2 b}{\sqrt{3}} A_{0}^{*} C_{2}+\frac{2 b}{\sqrt{3}} B_{1} O_{1}+d A_{0}^{2} O_{1}+2 d\left|A_{0}\right|^{2} B_{1}+B_{1}+\cdots\right) \\
& =\left(\frac{193 b d \sqrt{3}}{2^{5} 3^{4} q_{0}^{8}}-\frac{631 \sqrt{3} b^{3}}{3^{8} 2^{4} q_{0}^{12}}\right)\left|A_{0}\right|^{2} A_{0}^{2}-\frac{\sqrt{3}}{27 q_{0}^{4}} A_{0}^{2}+\text { o.t. },
\end{aligned}
$$

where o.t. stands for the other terms that I do not need. Retaining also only those terms that can give rise to powers of $A_{0}$ and $A_{0}^{*}$, the solvability condition for $A_{2}$ is, according to Eq. (D11), of the form

$$
\begin{aligned}
\partial_{T} A_{2}= & \frac{\partial A_{2}}{\partial \bar{X}^{2}}+A_{2}+\frac{2 b}{\sqrt{3}}\left(A_{0} O_{3}+A_{0}^{*} B_{3}\right) \\
& +d\left(A_{0}^{* 2} C_{2}+O_{1}^{2} A_{0}+2\left|B_{1}\right|^{2} A_{0}+2 B_{1} O_{1} A_{0}^{*}\right) \\
& -\frac{10}{9} e_{1}\left|A_{0}\right|^{4} A_{0}+\text { o.t. }
\end{aligned}
$$

Upon substitution of the appropriate expressions, the terms proportional to $\mathrm{O}_{3}$ and $\mathrm{B}_{3}$ also generate terms of the form $\left|A_{0}\right|^{2} A_{0}$, which correspond to a term $\epsilon k_{7}|\Phi|^{2} \Phi$ term in Eq. (6.4). Upon collecting the terms of the type $\left|A_{0}\right|^{4} A_{0}$, we finally get for $c_{2}$ in Eq. (6.4)

$$
c_{2}=\frac{10}{9} e_{1}-\frac{d^{2}}{192 q_{0}^{4}}+\frac{150679 b^{4}}{52488 q_{0}^{12}}-\frac{13903 b^{2} d}{7776 q_{0}^{8}} .
$$

In the case $b=0, e=1, q_{0}=1$, we thus get in the limit $d \rightarrow 0, \quad c_{2}=\frac{10}{9}, \quad$ while for $q_{0}=1, \quad e=0, \quad d=-1$, $b^{2}=27 / 38$, we get $c_{2}=2.714$. If we ignore for the latter set of parameters, the fact that $k_{4}$ is nonzero, and use the estimate $\epsilon c_{2} / c_{1}^{2}=\frac{3}{4}$ for the transition to nonlinear marginal stability, we get from this and Eq. (D18) 


$$
b(\epsilon) \approx 27 / 38+0.802 \sqrt{\epsilon} .
$$

This result is plotted in Fig. 15 with a dashed line.

I finally give an idea of the magnitude of the $k_{4}|\Phi|^{2}(\partial \Phi / \partial x)$ term in (6.4) in the regime where we expect the transition to nonlinear marginal stability, i.e., for $\epsilon c_{2} / c_{1}^{2} \simeq \frac{3}{4}$. To do so, we compare the magnitude of this term to the terms $c_{1}|\Phi|^{2} \Phi-c_{2}|\Phi|^{4} \Phi$ for the exact solution (4.12) of the real equation (6.1). Taking $\epsilon c_{2} / c_{1}^{2}=\frac{3}{4}$, we get with (4.12)

$$
\begin{aligned}
\frac{k_{4} \phi^{2} \partial \phi / \partial x}{c_{1} \phi^{3}-c_{2} \phi^{5}} & =\frac{k_{4} \epsilon^{1 / 2}\left(c_{1} \phi-2 c_{2} \phi^{3} / 3\right)}{c_{1}\left(c_{1} \phi-c_{2} \phi^{3}\right)} \\
& <\frac{k_{4} \epsilon^{1 / 2}}{c_{1}}=k_{4} \sqrt{3 / 4 c_{2}}=0.074 .
\end{aligned}
$$

Thus, although the effect of the complex term $i k_{4}|\Phi|^{2} \partial \Phi / \partial x$ will lead to a phase shift in $\Phi$, I originally expected that the threshold to nonlinear marginal stability is given rather accurately by (D23) for the value of $k_{4}$ found here in (D18).
${ }^{1}$ D. G. Aronson and H. F. Weinberger, in Partial Differential Equations and Related Topics, edited by J. A. Goldstein (Springer, Heidelberg, 1975); Adv. Math. 30, 33 (1978).

${ }^{2}$ G. Dee and J. S. Langer, Phys. Rev. Lett. 50, 383 (1983).

${ }^{3}$ E. Ben-Jacob, H. R. Brand, G. Dee, L. Kramer, and J. S. Langer, Physica 14D, 348 (1985). See also J. S. Langer, in Chance and Matter, edited by J. Souletie, J. Vannimenus, and R. Stora (North-Holland, Amsterdam, 1987).

${ }^{4}$ K. Nozaki and N. Bekki, Phys. Rev. Lett. 51, 2171 (1983).

${ }^{5}$ G. Dee, J. Stat. Phys. 39, 705 (1985); Physica 15D, 295 (1985).

${ }^{6}$ P. Collet and J.-P. Eckmann, Commun. Math. Phys. 107, 39

(1986); Helv. Phys. Acta 60, 969 (1987); Physica 140A, 96 (1986).

${ }^{7}$ V. G. Kamenskii and S. V. Manakov, Pis'ma Zh. Eksp. Teor. Fiz. 45, 499 (1987) [JETP Lett. 45, 638 (1987)]; (unpublished).

${ }^{8}$ B. Shraiman and D. Bensimon, Phys. Scr. T 9, 123 (1985).

${ }^{9}$ W. van Saarloos, Phys. Rev. Lett. 58, 2571 (1987); Phys. Rev. A 37, 211 (1988).

${ }^{10}$ G. Dee and W. van Saarloos, Phys. Rev. Lett. 60, 2641 (1988).

${ }^{11}$ G. Ahlers and D. S. Cannell, Phys. Rev. Lett. 50, 1583 (1983).

${ }^{12}$ M. Lücke, M. Mihelcic, and K. Wingerath, Phys. Rev. Lett. 52, 635 (1984); Phys. Rev. A 31, 396 (1985); M. Lücke, M. Mihelcic, and B. Kowalski, ibid. 35, 4001 (1987); M. Lücke, M. Mihelcic, B. Kowalski, and K. Wingerath, in The Physics of Structure Formation: Theory and Simulation, edited by W. Güttinger and G. Dangelmayr (Springer, New York, 1987).

${ }^{13}$ J. Fineberg and V. Steinberg, Phys. Rev. Lett. 58, 1332 (1987).

${ }^{14}$ L. Leger, Solid State Commun. 11, 1499 (1972), Mol. Cryst. Liq. Cryst. 24, 33 (1973); X. Y. Wang, Phys. Lett. A 112, 402 (1985); Phys. Rev. A 32, 3126 (1985); 34, 5179 (1986); L. Lin and Ch.-Q. Shu, Phys. Lett. A 119, 178 (1986); X. Yu-Zang and O.-Y. Zhong-can, Phys. Rev. A 37, 5002 (1988).

${ }^{15}$ G. Zhu, Phys. Rev. Lett. 49, 1332 (1982); X. Y. Wang, Phys. Lett. A 98, 259 (1983); Commun. Theor. Phys. 2, 1307 (1983); L. Lin, Ch.-Q. Shu, and X. Gang, J. Stat. Phys. 39, 633 (1985).

${ }^{16}$ P. E. Cladis, H. R. Brand, and P. L. Finn, Phys. Rev. A 28 , 512 (1983); J. E. Maclennan, M. A. Hanschy, and N. A. Clark, ibid. 34, 3554 (1986); see also P. E. Cladis and W. van Saarloos, in Solitons in Liquid Crystals, edited by L. Lam and J. Prost (Springer, New York, in press).

${ }^{17}$ A. Hanna, A. Saul, and K. Showalter, J. Am. Chem. Soc. 104, 3838 (1982); A. Saul and K. Showalter, in Oscillations and Traveling Waves in Chemical Systems, edited by R. J. Field and M. Burger (Wiley, New York, 1985).

${ }^{18} \mathrm{~A}$ very nice overview which also provides an introduction to the extensive Russian literature on the subject is given by $\mathrm{V}$. A. Vasiliev, Yu. M. Romanovskii, D. S. Chernavskii, and V. G. Yakhno, Autowave Processes in Kinetic Systems (Reidel, Dordrecht, 1987); see also P. Fife, in Nonequilibrium
Cooperative Phenomena in Physics and Related Fields, edited by M. G. Velarde (Plenum, London, 1984).

${ }^{19}$ J. P. Keener, SIAM J. Appl. Math. 39, 528 (1980); J. J. Tyson and J. P. Keener, Physica D 32, 327 (1988).

${ }^{20}$ R. J. Deissler, Phys. Lett. A 120, 334 (1987); Physica 25D, 233 (1987).

${ }^{21}$ R. J. Deissler and K. Kaneko, Phys. Lett. A 119, 397 (1987).

${ }^{22}$ See, for a review, A. Bers, in Handbook of Plasma Physics, edited by M. N. Rosenbluth and R. Z. Sagdeev (NorthHolland, Amsterdam, 1983), Vol. 1; a review with more emphasis on the applications in fluid mechanics is given by $\mathbf{P}$. Huerre, in Instabilities and Nonequilibrium Structures, edited by E. Tirapegui and D. Villarroel (Reidel, Dordrecht, 1987); and in Propagation in Systems Far from Equilibrium, edited by J. E. Wesfreid, H. R. Brand, P. Manneville, G. Albinet, and N. Boccara (Springer, New York, 1988).

${ }^{23}$ G. S. Triantafyllou, K. Kupfer, and A. Bers, Phys. Rev. Lett. 58, 1914 (1987).

${ }^{24}$ P. Huerre and P. A. Monkewitz, J. Fluid Mech. 159, 151 (1985).

${ }^{25}$ P. A. Monkewitz, Phys. Fluids 31, 999 (1988); P. A. Monkewitz and L. N. Nguyen, J. Fluids Struct. 1, 165 (1987).

${ }^{26}$ M. C. Cross, Phys. Rev. Lett. 57, 2935 (1986); Phys. Rev. A 38, 3593 (1988).

${ }^{27}$ J. Fineberg, E. Moses, and V. Steinberg, Phys. Rev. A 38 , 4939 (1988) and references therein.

${ }^{28}$ J. M. Chomaz, P. Huerre, and L. G. Redekopp, Phys. Rev. Lett. 60, 25 (1988).

${ }^{29}$ E. M. Lifshitz and L. P. Pitaevskii, Physical Kinetics, Course of Theoretical Physics (Pergamon, New York, 1981), Vol. 10, Chap. VI.

${ }^{30} \mathrm{~W}$. van Saarloos, Comments Condensed Matter Phys. (to be published).

${ }^{31}$ P. E. Cladis et al. (unpublished).

${ }^{32}$ M. Bramson, Mem. Am. Math. Soc. 285, 1 (1983).

${ }^{33}$ In Ref. 9, a factor $q^{r}$ [equivalent to the term $q^{i}$ in Eq. (2.3) of this paper] is missing, and as a result the right-hand sides of Eqs. (3.28)-(3.31) should be multiplied by $k^{r}$. This does not affect the conclusion of the analysis, however. I thank R. C. Aldredge III, for pointing this out to me.

${ }^{34}$ It is crucial here that a kink and antikink in equations of the form (3.3) attract each other. If this were not so, the second domain wall would not necessarily destroy the front. The presence of the attractive interaction is probably also the reason that the crossover to nonlinear marginal stability happens at a larger value of $b$ than the one estimated by putting $v_{w}=v^{*}=2$.

${ }^{35}$ See, e.g., A. Saul and K. Showalter, Ref. 17, p. 419; M. Otwinowski, P. Paul, and W. G. Laidlaw, Phys. Lett. A 128, 
483 (1988); X. Y. Wang, Phys. Lett. A 131, 277 (1988).

${ }^{36}$ The results discussed above for Eq. (4.1) are most easily derived in a similar way. See, e.g., A. Saul and K. Showalter, Ref. 17, p. 419.

${ }^{37}$ Note that all equations of the type (3.3) with $F(\phi)$ an odd fifth-order polynomial that has two stable states $\phi= \pm \phi_{s}$ can, by proper rescaling of $\phi$, be reduced to Eq. (4.7).

${ }^{38}$ See Ref. 9, Sec. III C for further details on the importance of initial conditions. In the present case, "sufficiently localized" means $\phi(x, t=0)<A \exp (-x)$ for $d \leq 2 / \sqrt{3}$ and $\phi(x, t=0)$ $<A \exp \left(-\phi_{s}^{2} x / \sqrt{3}\right)$ for $d>2 / \sqrt{3}$.

${ }^{39}$ J. Swift and P. C. Hohenberg, Phys. Rev. A 15, 319 (1977).

${ }^{40}$ See, e.g., P. C. Hohenberg and M. C. Cross, in Fluctuations and Stochastic Phenomena in Condensed Matter, edited by L. Garrido (Springer, New York, 1987).

${ }^{41}$ See, e.g., J. Guckenheimer and P. Holmes, Nonlinear Oscillations, Dynamical Systems and Bifurcations of Vector Field (Springer, New York, 1983).

${ }^{42}$ Although these are less relevant since they will be difficult to realize experimentally, $I$ also studied front propagation into the unstable state $\phi^{2}=\frac{1}{2}\left(c_{1} / c_{2}\right)^{1 / 2}\left[1-\left(1+4 \epsilon c_{2} / c_{1}^{2}\right)^{1 / 2}\right]$ for $\epsilon<0$. I have been unable to determine the crossover to nonlinear marginal stability for these fronts analytically along the lines sketched in Sec. IV. With the aid of numerical studies, I conclude that these fronts are governed by linear marginal stability with velocity $v^{*}=2 c_{1}\left[-1-4 \epsilon c_{2} / c_{1}^{2}+(1\right.$ $\left.\left.+4 \epsilon c_{2} / c_{1}^{2}\right)^{1 / 2}\right]^{1 / 2} / \sqrt{c_{2}}$ in the interval $-0.2416 \lesssim \epsilon c_{2} / c_{1}^{2}$ $\lesssim-0.19$ and by nonlinear marginal stability outside this range. Our estimate for the tiny interval $-0.25 \leq \epsilon c_{2} / c_{1}^{2} \lesssim-0.2416$, in which the front speed will be given by nonlinear marginal stability, is obtained as follows. For $\epsilon c_{2} / c_{1}^{2} \rightarrow-\frac{1}{4}$, the front speed should approach the limit $v=c_{1} / \sqrt{3 c_{2}}$ given by Eq. (6.3), whereas $v^{*} \rightarrow 0$ at this point. The crossover to nonlinear marginal stability must therefore occur approximately at the point where $v^{*}$ given by $(6.4)$ is equal to this value. Solving for the point where this happens yields the above estimate.

${ }^{43}$ While according to $(6.3)$ the interface between the two stable states does not move only for $\epsilon c_{2} / c_{1}^{2}=-\frac{3}{16}$, nonadiabatic effects can pin the interface in a finite range. See Y. Pomeau, Physica 23D, 3 (1986) and D. Bensimon, B. I. Shraiman, and V. Croquette, Phys. Rev. A 38, 5461 (1988).

${ }^{44}$ Although we consider in (6.4) only stationary bifurcations to facilitate the direct comparison with Eq. (6.1), a qualitatively similar crossover from linear to nonlinear marginal stability will hold in systems that exhibit convective instabilities. See Sec. $\mathrm{X}$ for some possible implications of this.

${ }^{45}$ M. C. Cross, P. G. Daniels, P. C. Hohenberg, and E. D. Siggia, J. Fluid Mech. 127, 155 (1983). See, in particular, Appendix F.

46J. J. Tyson and P. C. Fife, J. Chem. Phys. 73, 2224 (1980).

${ }^{47}$ That the velocities of fronts into an unstable state $\phi_{U S}$ and into a metastable state $\phi_{\mathrm{MS}}$ should approach the same limit at the point where these two states coalesce can be seen as follows. The equation for steady-state profiles $\phi(x-v t)$ for Eq. (3.3) is of the form of the equation of motion for the particle in a potential with friction, $d^{2} \phi / d x^{2}=-v d \phi / d x-d V / d \phi$, with $V=\int d \phi F(\phi)$. Close to the point where an unstable and a metastable state coalesce, the front velocity into the unstable state is always given by nonlinear marginal stability, as the criterion for nonlinear marginal stability $v^{\dagger}$ is equivalent to the requirement that the particle with this "friction" $v^{\dagger}$ approach the minimum of $V$ corresponding to $\phi_{\mathrm{US}}$ without overshooting it (since $v^{*}$ becomes arbitrarily small when $\phi_{\mathrm{US}} \rightarrow \phi_{\mathrm{MS}}$, the "friction" $v^{*}$ always leads to an overshoot in this limit). The velocity $v_{w}$ of a front or wall into the nearly metastable state $\phi_{\mathrm{ms}}$ corresponds to the value of the "friction" such that the particle just makes it to the nearly local maximum. Physically, it is then clear that $v^{\dagger} \rightarrow v_{w}$ when $\phi_{\mathrm{US}} \rightarrow \phi_{\mathrm{MS}}$.

${ }^{48}$ C. M. Bender and S. A. Orszag, Advanced Mathematical Methods for Scientists and Engineers (McGraw-Hill, New York, 1978), p. 11.

${ }^{49}$ See, e.g., G. B. Whitham, Linear and Nonlinear Waves (Wiley, New York, 1974).

${ }^{50}$ This fact was also stressed to me by D. A. Kurtze.

${ }^{51}$ In fact, if we analyze Eq. (9.6) at an extremum where $k=k_{1}$ in practice there will be one zero mode at $Q=-2 k_{1}^{r}$. To see this, note that the linear perturbation $e^{i k_{1} x}+A e^{-k_{i}^{i} x-i k_{1}^{r} x}$ is clearly a zero mode for $\phi$. Writing this as $\exp \left[i k_{1} x\left(1+A e^{-2 i k_{1}^{r} x}\right)\right]$ shows that $e^{-2 i k_{1}^{r} x}$ must be a zero mode of $\mathcal{L}$. See Ref. 9 for explicit examples.

${ }^{52}$ W. Schöpf and W. Zimmermann, Europhys. Lett. 8, 41 (1989), and references therein.

${ }^{53}$ Note that it is hard to understand why the crossover speed in the experiments of Ref. 27 appears to be less than $v^{*}$, just as it is hard to understand why the front speeds measured by Ahlers and Cannell, Ref. 11, are less than $v^{*}$.

${ }^{54}$ T. von Kármán, Göttinger Nachr. Math. Phys. Klasse (1911), pp. 509-517.

${ }^{55}$ C. Mathis, M. Provansal, and L. Boyer, J. Phys. (Paris) Lett. 45, 483 (1984); M. Provansal, in Propagation in Systems Far from Equilibrium, edited by J. E. Wesfreid, H. R. Brand, P. Manneville, G. Albinet, and N. Boccara (Springer, New York, 1988).

${ }^{56}$ S. J. Davies and C. M. White, Proc. R. Soc. London, Ser. A 119, 92 (1928); T. W. Kao and C. Park, J. Fluid Mech. 43, 145 (1970).

${ }^{57}$ L. H. Thomas, Phys. Rev. 91, 780 (1953).

${ }^{58}$ R. J. Deissler, Phys. Fluids 30, 2303 (1987).

${ }^{59}$ D. Meksyn and J. T. Stuart, Proc. R. Soc. London, Ser. A 208, 517 (1951).

${ }^{60}$ K. Stewartson and J. T. Stuart, J. Fluid Mech. 48, 529 (1971).

${ }^{61}$ S. A. Orszag and L. C. Kells, J. Fluid Mech. 96, 159 (1980).

${ }^{62}$ See, e.g., T. Herbert, Fluid Dyn. Trans. 11, 77 (1983). 